\title{
Evolutionary Bioinformatics
}

\section{Prediction of Protein Essentiality by the Support Vector Machine with Statistical Tests}

\author{
Chiou-Yi Hor, Chang-Biau Yang, Zih-Jie Yang and Chiou-Ting Tseng \\ Department of Computer Science and Engineering, National Sun Yat-sen University Kaohsiung 80424, Taiwan. \\ Corresponding author email: cbyang@cse.nsysu.edu.tw
}

\begin{abstract}
Essential proteins include the minimum required set of proteins to support cell life. Identifying essential proteins is important for understanding the cellular processes of an organism. However, identifying essential proteins experimentally is extremely timeconsuming and labor-intensive. Alternative methods must be developed to examine essential proteins. There were two goals in this study: identifying the important features and building learning machines for discriminating essential proteins. Data for Saccharomyces cerevisiae and Escherichia coli were used. We first collected information from a variety of sources. We next proposed a modified backward feature selection method and build support vector machines (SVM) predictors based on the selected features. To evaluate the performance, we conducted cross-validations for the originally imbalanced data set and the down-sampling balanced data set. The statistical tests were applied on the performance associated with obtained feature subsets to confirm their significance. In the first data set, our best values of F-measure and Matthews correlation coefficient (MCC) were 0.549 and 0.495 in the imbalanced experiments. For the balanced experiment, the best values of F-measure and MCC were 0.770 and 0.545 , respectively. In the second data set, our best values of F-measure and MCC were 0.421 and 0.407 in the imbalanced experiments. For the balanced experiment, the best values of F-measure and MCC were 0.718 and 0.448 , respectively. The experimental results show that our selected features are compact and the performance improved. Prediction can also be conducted by users at the following internet address: http://bio2.cse.nsysu.edu.tw/ esspredict.aspx.
\end{abstract}

Keywords: support vector machine, feature selection, protein-protein interaction, essential protein, statistical test

Evolutionary Bioinformatics 2013:9 387-416

doi: $\underline{10.4137 / E B O . S 11975}$

This article is available from http://www.la-press.com.

(c) the author(s), publisher and licensee Libertas Academica Ltd.

This is an open access article published under the Creative Commons CC-BY-NC 3.0 license. 


\section{Introduction}

Identifying essential proteins is important for understanding the cellular processes in an organism because no other proteins can perform the functions of essential proteins. Once an essential protein is removed, dysfunction or cell death results. Thus, several studies have been conducted to identify essential proteins. Experimental approaches for identifying essential proteins include gene deletion, ${ }^{1}$ RNA interference, ${ }^{2}$ and conditional knockouts. ${ }^{3}$ However, these methods are labor-intensive and time-consuming. Hence, alternative methods for identifying essential proteins are necessary.

The essential protein classification problem involves determining the necessity of a protein for sustaining cellular function or life. Among the methods available for identifying essential proteins, machinelearning based methods are promising approaches. Therefore, several studies have been conducted to examine the effectiveness of this technique. $\mathrm{Chin}^{4}$ proposed a double-screening scheme and constructed a framework known as the hub analyzer (http://hub.iis. sinica.edu.tw/Hubba/index.php) to rank the proteins. Acencio and Lemke ${ }^{5}$ used Waikato Environment for Knowledge Analysis (WEKA) ${ }^{6}$ to predict the essential proteins. Hwang et $\mathrm{al}^{7}$ applied a support vector machine (SVM) to classify the proteins.

Protein-protein interactions (PPIs) are well-known to be significant characteristics of protein function. Several studies have attempted to predict and classify protein function ${ }^{8}$ as well as analyze protein phenotype ${ }^{9}$ by studying interactions. A previous study ${ }^{10}$ further suggested that essential proteins and nonessential proteins can be discriminated by means of topological properties derived from the PPI network. In spite of the above superior properties, however, analyzing PPI experimentally is time-consuming. With the advent of yeast two-hybrid ${ }^{11}$ high-throughput techniques, which can be used to identify several PPIs in one experiment, obtaining PPI information has become easier. Since a PPI network is similar to a social network in many aspects, some researchers apply social network techniques for analyzing PPI networks. Thus, several topological properties have been extensively explored and studied in recent years.

Fundamental properties, such as sequence or protein physiochemical ones, are not subjected to detailed examination in previous studies. This may be because each of these preliminary properties alone is somewhat less relevant to essentiality. However, this information is highly accessible because only sequence information is required to derive these properties. Hence, we included these properties in our study. For topological properties, in addition to physical interactions, we incorporated a variety of interaction information, including metabolic, transcriptional regulation, integrated functional, and genomic context interactions. Our experimental results revealed that these features provide either complement information for essentiality identification or provide other biological justification.

To identify the reduced feature subset, which is crucial for biological processes, previous studies have used feature selection techniques. The advantages of this method include storage reduction, performance improvement or data interpretation. ${ }^{12}$ In accordance with whether the feature selection procedure is bound with the predictor, the method is roughly classified into three categories: filter, wrapper, and embedded. Filter methods often provide a complete order of available features in terms of relevance measures. Methods such as Fisher score, ${ }^{12}$ mutual information, minimal redundancy and maximal relevance (mRMR), ${ }^{13}$ conditional mutual information maximization (CMIM) ${ }^{14}$ and minimal relevant redundancy $(\mathrm{mRR})^{15}$ belong to this category. Both wrapper and embedded methods involve the selection process as a part of the learning algorithm. The former utilizes a learning machine to evaluate subsets of features according to some performance measurements. For example, sequential backward and forward feature selection ${ }^{12}$ falls into this category. Embedded methods directly perform feature selection in the learning process and they are usually specific to given learning machines. Example include C4.5, ${ }^{16}$ Classification and Regression Trees (CART),${ }^{17}$ and ID3. ${ }^{18}$ Additionally, some researchers proposed an information-gain based the feature selection method, ${ }^{19}$ which examines the effectiveness of classifier combination.

In this paper, we used two datasets. The first one was from Saccharomyces cerevisiae. The corresponding PPI data set was Scere20070107, which was obtained from the DIP database. The data set totally contains 4873 proteins and 17,166 interactions. Our feature set consisted of the features obtained or extracted from 
the methods proposed by Acencio and Lemke, ${ }^{5} \mathrm{Chin},{ }^{4}$ Hwang et al, ${ }^{7}$ and Lin et al. ${ }^{20}$ The second data set was from Escherichia coli, which was first compiled by Gustafson et al. ${ }^{21}$ The data set totally contained 3569 proteins. The associated network information included physical, integrated functional, and genomic context interactions and is collected from $\mathrm{Hu}$ et al. ${ }^{22}$ For both data sets, we propose a modified sequential backward feature selection method for selecting important features.

Next, SVM models were built using the selected feature subsets. In this study, the SVM software LIB$\mathrm{SVM}^{23}$ was adopted for classification models. Each model was applied to both imbalanced and balanced data sets. The results were compared with those of previous studies and statistical tests were conducted to examine significance. For the imbalanced $S$. cerevisiae data, our best results for F-measure and MCC were 0.549 and 0.495 , respectively, which outperform the best previous method ${ }^{7}$ with results of 0.354 and 0.36 , respectively. We obtained values of 0.770 and 0.545 for F-measure and $\mathrm{MCC}$ in the balanced data experiment, which was superior to the best previous $\operatorname{method}^{7}$ with 0.737 and 0.492 , respectively. For experiments examining the $E$. coli data set, our best values for F-measure and MCC were 0.421 and 0.407 , respectively, in the imbalanced data set. In the balanced experiment, the best values for F-measure and MCC were 0.718 and 0.448 , respectively. The results are similar to those of Gustafson et al, ${ }^{21}$ who examined 29 features, but in our method, only five or seven features were used for prediction. To verify whether our improvement was statistically significant, we performed bootstrap crossvalidation ${ }^{24}$ on performance measures.

\section{Background}

\section{The data set}

In this paper, we used two data sets for experiments: $S$. cerevisiae and E. coli. The former included PPI network data. We downloaded the data set from the DIP (http://dip.doe-mbi.ucla.edu/) website. ${ }^{25}$ The original data set contained 4873 proteins and 17166 interactions. To comply with previous studies, we also adopted the largest connected component of the network data. There were a total of 4815 proteins, including 975 essential proteins and 3840 nonessential proteins. The information of protein essentiality was obtained from the Saccharomyces Genome
Database (SGD), which is located at http://www. yeastgenome.org/. Since this data set has been used in several previous studies, we thus obtained and incorporated various related features for experiments.

The E. coli data set was obtained from Gustafson et al. ${ }^{21}$ It contained 3569 proteins, among which 611 are essential. Due to availability and coverage issues, we used another information from three additional networks: physical interaction (PI), integrated functional interaction, and integrated PI and genomic context (GC) network. The information was collected from $\mathrm{Hu}$ et al. ${ }^{22}$

In the above two data sets, the ratio of nonessential proteins to essential proteins was approximately $4: 1$ and 5:1, respectively. The data imbalance will inevitably led to biased fitting to nonessential proteins during the learning processes. Thus, we constructed another balanced data set. Taking the first data set, for example, we randomly selected 975 nonessential proteins and mixed them with essential proteins to form a balanced data set. In the new data set, the number of nonessential data elements against that of essential elements was equal.

\section{Bootstrap cross-validation}

We used bootstrap cross-validation (BCV) to compare the performance of the two classifiers using the $k$-fold crossvalidation. Assume that a sample $S=\left\{\left(\mathrm{x}_{1}, y_{1}\right),\left(\mathrm{x}_{2}, y_{2}\right), \ldots\right.$, $\left.\left(\mathrm{x}_{\mathrm{n}}, y_{n}\right)\right\}$ is composed of $n$ observations, where $\mathrm{x}_{\mathrm{i}}$ represents the feature vector of the $i$ th observation and $y_{i}$ denotes the class label associated with $\mathrm{x}_{\mathrm{i}}$. A bootstrap sample $S_{b}^{*}=\left\{\left(\mathrm{x}_{1}^{*}, y_{1}^{*}\right),\left(\mathrm{x}_{2}^{*}, y_{2}^{*}\right), \ldots,\left(\mathrm{x}_{\mathrm{n}}^{*}, y_{n}^{*}\right)\right\}$ consists of $n$ observations that are sampled from $S$ with replacement, where $1 \leq b \leq B$, and $B$ is a constant between 50 and 200. For each sample $S_{b}^{*}$, a $k$-fold cross-validation was carried out. The performance measure $c_{b}$, such as error rate, was calculated with $S_{b}^{*}$. The procedure was repeated $B$ times and then the average performance measure $C_{B}=\sum_{\mathrm{b}=1}^{\mathrm{B}} c_{b} / B$ was evaluated over the $B$ bootstrap samples. Since the distribution of the bootstrap performance measures was approximately normal, the confidence interval and significance were estimated accordingly.

\section{Performance measures}

In this study, the performance measures included precision, recall, F-measure (F1), Matthews correlation coefficient (MCC), and top percentage 
of essential proteins. Their formulas are given as follows:

1. Precision: $T P / T P+F P$

2. Recall: $T P / T P+F N$

3. F-measure: $2 \times$ precision $\times$ recall $/($ precision + recall $)$

4. $\mathrm{MCC}: \frac{T P \times T N-F P \times F N}{\sqrt{(T P+F N)(T P+F P)(T N+F P)(T N+F N)}}$

5. Top percentage of essential protein: Number of real essential proteins in top $n$. $n$

Here, an essential protein is represented by the positive observation. True positive (TP), true negative (TN), false positive (FP), and false negative (FN) represent the numbers of true positive, true negative, false positive, and false negative proteins, respectively. The value $\mathrm{n}$ denotes the total number of predictions. In addition, receiver operating characteristic (ROC) curve $\mathrm{e}^{18}$ and area under curve (AUC) were used to evaluate the classification performance.

\section{Feature extraction}

The feature set we used included sequence properties (S), such as amino acid occurrence and average amino acid PSSM; protein properties (P), such as cell cycle and metabolic process; topological properties $(\mathrm{T})$, such as bit string of double screening scheme and betweenness centrality related to physical interactions; and other properties $(\mathrm{O})$, such as phyletic retention and essential index. There were a total of 45 groups and 90 features in the $S$. cerevisiae data set. For the E. coli data set, there were 35 groups and 80 features. All names and sources are shown in Table 1. Only Bit string of double screening scheme is presented.

The remaining features are detailed in the Appendix.

Lin et $\mathrm{al}^{26}$ and $\mathrm{Chin}^{4}$ proposed the double screening scheme. They used multiple ranking scores to sort essential proteins. The drawback is that each protein does not have a unique score. Thus, we propose a bit string implementation to incorporate these two properties into a single score.

An example of our bit string implementation is shown in Tables 2 and 3. Suppose that four proteins, $\mathrm{W}, \mathrm{X}, \mathrm{Y}$, and $\mathrm{Z}$, are to be ranked. In the first itera- tion, we desire to find the top one protein. We first select the top 2 proteins using the ranking method A, which are $\mathrm{W}$ and $\mathrm{X}$. Next, we use method $\mathrm{B}$ to rank these two proteins. The ranks of $\mathrm{W}$ and $\mathrm{X}$ are 2 and 1 , respectively. Hence, in the first iteration, $\mathrm{X}$ is finally selected. It follows the bit $\mathrm{M}[\mathrm{X}, 1]$ is set to 1 , and others, $\mathrm{M}[\mathrm{W}, 1], \mathrm{M}[\mathrm{Y}, 1]$ and $\mathrm{M}[\mathrm{Z}, 1]$, are set to 0 . In the second iteration, 2 top-ranking proteins are to be found. First, four proteins W, X, $\mathrm{Y}$ and $\mathrm{Z}$ are selected, because they are the top 4 proteins by ranking method $\mathrm{A}$. Next, with ranking method $B$, we select the top 2 proteins from them, which are $\mathrm{X}$ and $\mathrm{Y}$. Hence, the bits corresponding to $M[X, 2]$ and $M[Y, 2]$ are set to 1 , and the others are set to 0 in this iteration. Finally, we sum up the bits of each protein, as shown in the fourth column of Table 3.

There is still an issue in the bit string implementation, that is, $\mathrm{M}$ may be too sparse to be handled by classifiers. Since the number of proteins being selected is around $n / 2$, the sum of about $n / 2$ bits is close to 0 . In our experience, this makes it difficult to distinguish between proteins. To overcome this problem, for each protein, we added another score $n-r$ to the sum of the bit string, where $r$ is the rank of the protein by the ranking method B. In this study, we used DMNC to rank A and MNC to rank B. In this example, $n=4$, so the values $n-r$ of $W, X, Y$ and $Z$ are $0,2,3$, and 1 , respectively. We summed the values with the bit string; hence, the final scores are $0,4,4$, and 1 . The overall procedure is given in the Procedure bit string implementation of DSS.

\section{Sequential backward feature selection method}

SVM is a well-established tool for data analysis which has been shown to be useful in various fields, such as text summarization, ${ }^{27}$ intrusion detection, ${ }^{28}$ and image coding. ${ }^{29}$ In this study, we utilized the SVM software developed by Chang and Lin, called LIBSVM. ${ }^{23}$ To address the data imbalance, we propose the modified sequential backward feature selection method.

Since most data were nonessential, choosing only accuracy as an objective or adopting conventional feature ranking schemes favored negative data. As more and more features were excluded, overall accuracy declined. Since the number of negative data elements 
Table 1. Protein features.

\begin{tabular}{|c|c|c|c|c|c|c|}
\hline ID & Property name & Type & Size & Sub-names & S. cere & E. coli \\
\hline 1 & Amino acid occurrence ${ }^{20}$ & S & 20 & $A \ldots Y$ & - & - \\
\hline 2 & Average amino acid PSSM²0 & $\mathrm{S}$ & 20 & $A \ldots Y$ & - & • \\
\hline 3 & Average cysteine position ${ }^{20}$ & $\mathrm{~s}$ & 1 & & - & - \\
\hline 4 & Average distance of every two cysteines ${ }^{20}$ & $\mathrm{~s}$ & 1 & & - & - \\
\hline 5 & Average hydrophobic ${ }^{20}$ & $\mathrm{~s}$ & 1 & & - & - \\
\hline 6 & Average hydrophobicity around cysteine ${ }^{20}$ & $\mathrm{~s}$ & 4 & $1 \ldots 4$ & - & • \\
\hline 7 & Cysteine count ${ }^{20}$ & $\mathrm{~s}$ & 1 & & • & - \\
\hline 8 & Cysteine location 20 & $\mathrm{~s}$ & 5 & $1 \ldots 5$ & - & - \\
\hline 9 & Cysteine odd-even index ${ }^{20}$ & $\mathrm{~S}$ & 1 & & • & • \\
\hline 10 & Protein length ${ }^{20}$ & $\mathrm{~s}$ & 1 & & - & - \\
\hline 11 & Cell cycle ${ }^{5}$ & $\mathrm{P}$ & 1 & & - & \\
\hline 12 & Cytoplasm $^{5}$ & $P$ & 1 & & - & \\
\hline 13 & Endoplasmic reticulum 5 & $\mathrm{P}$ & 1 & & - & \\
\hline 14 & Metabolic process $^{5}$ & $P$ & 1 & & - & \\
\hline 15 & Mitochondrion $^{5}$ & $P$ & 1 & & - & \\
\hline 16 & Nucleus $^{5}$ & $P$ & 1 & & - & \\
\hline 17 & Other process ${ }^{5}$ & $P$ & 1 & & - & \\
\hline 18 & Other localization ${ }^{5}$ & $P$ & 1 & & - & \\
\hline 19 & Signal transduction ${ }^{5}$ & $P$ & 1 & & - & \\
\hline 20 & Transport $^{5}$ & $\mathrm{P}$ & 1 & & - & \\
\hline 21 & Transcription $^{5}$ & $P$ & 1 & & - & \\
\hline 22 & Betweenness centrality related to all interactions ${ }^{41}$ & $\mathrm{~T}$ & 1 & & • & • \\
\hline 23 & Betweenness centrality related to metabolic interactions ${ }^{5}$ & $\mathrm{~T}$ & 1 & & • & \\
\hline 24 & Betweenness centrality related to physical interactions ${ }^{5}$ & $\mathrm{~T}$ & 1 & & • & • \\
\hline 25 & Betweenness centrality transcriptional regulation interactions ${ }^{5}$ & $\mathrm{~T}$ & 1 & & - & \\
\hline 26 & Bit string of double screening scheme [this paper] & $\mathrm{T}$ & 1 & & - & • \\
\hline 27 & Bottleneck 8,41 & $\mathrm{~T}$ & 1 & & $\bullet$ & $\bullet$ \\
\hline 28 & Clique level $^{7}$ & $\mathrm{~T}$ & 1 & & • & • \\
\hline 29 & Closeness centrality ${ }^{42}$ & $\mathrm{~T}$ & 1 & & - & • \\
\hline 30 & Clustering coefficient ${ }^{7}$ & $\mathrm{~T}$ & 1 & & - & - \\
\hline 31 & Degree related to all interactions ${ }^{43}$ & $\mathrm{~T}$ & 1 & & • & - \\
\hline 32 & Degree related to physical interactions ${ }^{5}$ & $\mathrm{~T}$ & 1 & & - & • \\
\hline 33 & Density of maximum neighborhood component ${ }^{4}$ & $\mathrm{~T}$ & 1 & & • & - \\
\hline 34 & Edge percolated component ${ }^{9}$ & T & 1 & & • & • \\
\hline 35 & Indegree related to metabolic interaction ${ }^{5}$ & $\mathrm{~T}$ & 1 & & • & \\
\hline 36 & Indegree related to transcriptional regulation ${ }^{5}$ & $\mathrm{~T}$ & 1 & & - & \\
\hline 37 & Maximum neighborhood component ${ }^{4}$ & $\mathrm{~T}$ & 1 & & - & • \\
\hline 38 & Neighbors' intra-degree ${ }^{7}$ & $\mathrm{~T}$ & 1 & & • & • \\
\hline 39 & Outdegree related to metabolic interaction ${ }^{5}$ & $\mathrm{~T}$ & 1 & & - & \\
\hline 40 & Outdegree related to transcriptional regulation interaction ${ }^{5}$ & $\mathrm{~T}$ & 1 & & - & \\
\hline 41 & Betweenness centrality related to integrated functional interaction ${ }^{22}$ & $\mathrm{~T}$ & 1 & & & • \\
\hline 42 & Betweenness centrality related to integrated PI and GC network ${ }^{22}$ & $\mathrm{~T}$ & 1 & & & • \\
\hline 43 & Degree related to integrated functional interaction ${ }^{22}$ & $\mathrm{~T}$ & 1 & & & - \\
\hline 44 & Degree related to integrated PI and GC network ${ }^{22}$ & $\mathrm{~T}$ & 1 & & & • \\
\hline 45 & Common function degree ${ }^{7}$ & 0 & 1 & & • & \\
\hline 46 & Essential index ${ }^{7}$ & $\mathrm{O}$ & 1 & & • & \\
\hline 47 & Identicalness ${ }^{5}$ & 0 & 1 & & • & \\
\hline 48 & Open reading frame length ${ }^{7}$ & $\mathrm{O}$ & 1 & & • & - \\
\hline 49 & Phyletic retention ${ }^{21}$ & $\mathrm{O}$ & 1 & & - & - \\
\hline 50 & Number of paralagous genes ${ }^{21}$ & $\mathrm{O}$ & 1 & & & • \\
\hline 51 & Codon Adaptation Index $(\mathrm{CAI})^{21,44}$ & $\mathrm{O}$ & 1 & & & - \\
\hline 52 & Codon Bias Index (CBI) & $\mathrm{O}$ & 1 & & & • \\
\hline
\end{tabular}

(Continued) 
Table 1. (Continued)

\begin{tabular}{|c|c|c|c|c|c|c|}
\hline ID & Property name & Type & Size & Sub-names & S. cere & E. coli \\
\hline 53 & Frequency of optimal codons ${ }^{21,44}$ & $\mathrm{O}$ & 1 & & & • \\
\hline 54 & Aromaticity score s1,44 $^{21}$ & $\mathrm{O}$ & 1 & & & - \\
\hline 55 & Leading strand of the circular chromosome ${ }^{21}$ & O & 1 & & & $\bullet$ \\
\hline & Total & & 100 & & 90 & 80 \\
\hline
\end{tabular}

Notes: S. cere and E. coli mean Saccharomyces cerevisiae and Escherichia coli datasets, respectively. For topological features, if not particularly mentioned, they are related to physical interactions. Due to coverage or availability issue, we adopt different features for $S$. cere and $E$. coli datasets. For example, interactions in E. coli data set contain integrated functional, PI, and GC network information while those in S. cere include metabolic, transcriptional regulation and PI network information.

Abbreviations: GC, genomic context; $\mathrm{PI}$, physical interactions

was higher than that of positive factors, the truepositive rate thus decreased more than the true-negative rate. Thus, features should be selected that most positive samples are correctly classified while not deteriorating the overall accuracy too much. In this sense, rather than using only accuracy to guide the feature selection, we used a composite score $C$ as the objective function. The composite score was represented in terms of precision $(\mathrm{P})$, recall $(\mathrm{R}), \mathrm{F}$-measure $(\mathrm{F})$, and MCC (M) and was given as $C=w_{P} * P+w_{R} * R+w_{F}$ $* F+w_{M} * M$. The four adjustable weights, $w_{P}, w_{R}, w_{F}$, and $w_{M}$, were used, leading to compromise among the associated performance measures. An additional punishment was imposed to $C$ to allow scores associated

\section{Procedure: Bit String Implementation of DSS}

input: $P$ : proteins for ranking, where $|P|=n$

$A$ : ranking methods, where $|A|=m=2$

$M$ : bit matrix of $n \times \frac{n}{2}$, initialized by 0

output: Protein ranking scores $R$

begin

for $i=1$ to $n$ do $R[i]=0$;

for $i=1$ to $\lfloor n / 2\rfloor$ do

$T_{1}=$ top $2 i$ proteins ranked by $A(1)$ in $P$;

$T_{2}=$ top $i$ proteins ranked by $A(2)$ in $T_{1}$;

foreach $x \in T_{2}$ do $M[x, i]=1$;

end

for $i=1$ to $n$ do

foreach $j=1$ to $\lfloor n / 2\rfloor$ do $R[i]=R[i]$

$+M[i, j]$

end

$\mathrm{T}=$ protein orders ranked by $\mathrm{A}(m)$;

foreach $i=1$ to $n$ do $R[i]=R[i]+(n-T[i])$ end with fewer features could compete with those with more features. That is,

$$
\begin{aligned}
C(S)= & w_{P} * P(S)+w_{R} * R(S)+w_{F} * F(S)+w_{M} \\
& * M(S) \times(|S|-t) * u(|S|-t) * e,
\end{aligned}
$$

where $S$ denotes the selected feature subset. $|S|$ and $t$ denote the size of $S$ and the goal number of features specified by a user, respectively. The unit step function $u(|S|-t)=0$ as $|S|-t \leq 0$, otherwise $u(|S|-t)=1$. Finally, a threshold $\rho$ was adopted to make ensure that the improvement over feature changes was not a random process. The value of $\rho$ was estimated by comparing average score difference among feature subsets of sizes $p$ and $p+1$ in the preliminary run for given several different values of $p$. The value $e$ denotes the penalty score when an additional feature is selected. The score is also specified by the user and should be slightly larger than $\rho$ to encourage feature subsets of smaller sizes. The procedure for feature selection is described in Procedure backward feature selection.

\section{Experimental procedure and results}

For comparison purposes, we used two feature selection methods: mRMR and CMIM. In the $S$. cerevisiae

Table 2. Ranking by two different methods, where smaller numbers indicate higher ranks.

\begin{tabular}{lll}
\hline Protein name & \multicolumn{2}{l}{ Ranking method } \\
\cline { 2 - 3 } & A (DMNC) & B (MNC) \\
\hline W & 1 & 4 \\
X & 2 & 2 \\
Y & 3 & 1 \\
Z & 4 & 3 \\
\hline
\end{tabular}




\section{Procedure: Backward Feature Selection}

input : $e$ : penalty score

$k$ : number of folds for cross-validation

$r$ : maximal number of rounds to retry

$S_{0}$ : set of all available features, where

$\left|S_{0}\right|=n$

$t$ : goal number of selected features

$w_{P}, w_{R}, w_{F}$ and $w_{M}$ : weights of performance measures

$\rho$ : minimal improvement to proceed

output: Selected feature subset $S$

data: $50 \%$ of all available data elements

begin

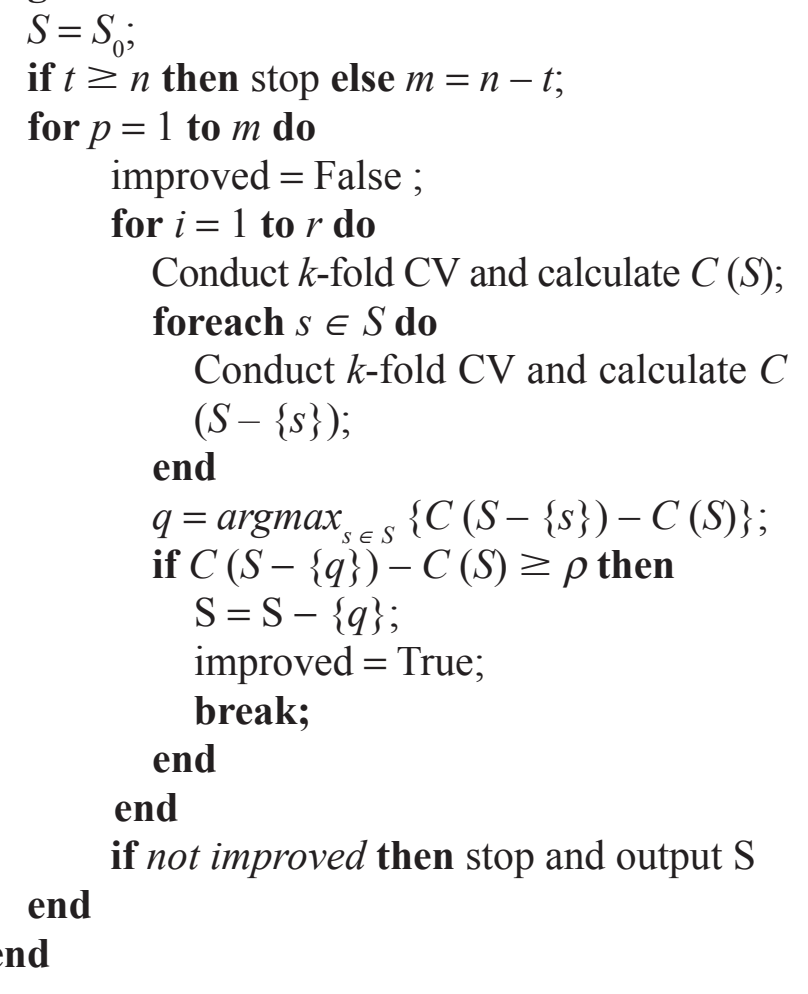

data set, our results were also compared to those of Acencio and Lemke ${ }^{5}$ and Hwang et al. ${ }^{7}$ For the E. coli data set, we also compared our results with those of Gustafson et al. ${ }^{21}$

\section{Experimental procedure}

The overall procedure of our experiments is illustrated in Figure 1 and is described as follows.

\section{Stage 1: Determine benchmark feature set}

For the $S$. cerevisiae data set, we used Hwang's feature set as the benchmark. For the $E$. coli data set, we
Table 3. Bit strings by the double screening method.

\begin{tabular}{llllll}
\hline $\begin{array}{l}\text { Protein } \\
\text { name }\end{array}$ & \multicolumn{2}{c}{$\boldsymbol{i}$ th iteration } & $\begin{array}{l}\text { Sum of bit } \\
\text { string }\end{array}$ & $\boldsymbol{n}-\boldsymbol{r}$ & Sum \\
\cline { 2 - 3 } & 1st & 2nd & & & \\
\hline W & 0 & 0 & 0 & 0 & 0 \\
X & 1 & 1 & 2 & 2 & 4 \\
Y & 0 & 1 & 1 & 3 & 4 \\
Z & 0 & 0 & 0 & 1 & 1 \\
\hline
\end{tabular}

used Gustafson's feature set as the benchmark. These two feature sets are considerably effective for various performance measures.

\section{Stage 2: Tune SVM parameters for best performance}

For the above two feature sets, we first ran the SVM software using the feature sets of Hwang or Gustafson and tuned the SVM parameters to achieve the highest average performances.

\section{Stage 3: Adopt best performances as reference performances}

After determining the best SVM parameters for the feature sets of Hwang and Gustafson, we recorded the SVM parameters and results. To compare our results with other models, such as those obtained using our methods, mRMR and CMIM, we used the same SVM software and adjust the cost parameters of SVM in order to achieve similar levels of precision.

\section{Stage 4: Perform feature selection}

We randomly chose $50 \%$ of available data. Next, the backward feature selection procedure was applied to these selected data. In the beginning of our feature selection procedure, we imposed no penalty on the score calculation. Hence, the procedure attempts to achieve the highest score. In the subsequent runs, we added penalties for feature sizes to the score calculation. Subsets with smaller feature size but only slightly inferior in

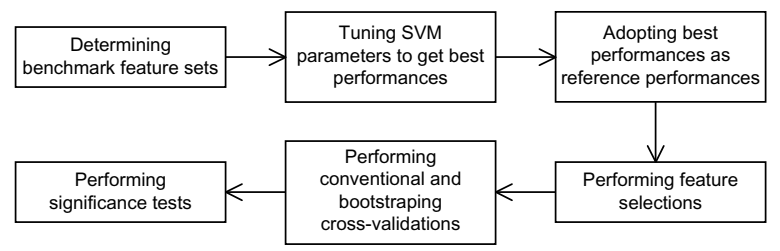

Figure 1. Flowchart for the construction of SVM models and performance comparison. 
performance were selected. To compare our results with those of other methods, we also used the mRMR and CMIM feature ranking methods and chose subsets as in Procedure backward feature selection.

\section{Stage 5: Perform 10-fold and bootstrap cross-validations}

The data were prepared in both balanced and imbalanced manners. For each data set, we randomly partitioned all data into 10 disjoint groups and used the feature subsets selected in the previous stage to calculate various performance measures. The data were prepared in both balanced and imbalanced manners. The 10 -fold cross validation was repeated 10 times and average performance measures were computed. Next, a bootstrap sampling procedure was conducted and 200 bootstrap samples were produced, including both balanced and imbalanced samples. Each bootstrap sample was also partitioned for 10 -fold cross validations and performance measures were calculated. Note that all models were examined by the same sets of data partitions for conventional and bootstraping cross-validations.

\section{Stage 6: Perform significance tests}

Once bootstrap cross validations were carried out, the significance tests were adopted accordingly. In addition to the average values of AUC, precision, recall, F-measure, and MCC, we conducted a statistical significance test for these performance measures. Additionally, we calculated ROC curves and top percentage values for imbalanced experiments.

\section{Backward feature selection and $\mathrm{mRMR} / \mathrm{CMIM}$ feature ranking}

We used $50 \%$ of available data elements for feature selection. Taking the $S$. cerevisiae data set as an example, only $(3840+975) \times 50 \%$ observations were randomly chosen for the Backward feature selection procedure. During the procedure, several performance scores were calculated by means of $k$-fold cross-validations. In the first run, the parameters were set as follows: $k=2, w_{P}=1, w_{R}=1, w_{F}=1$, $w_{M}=1, \rho=0.005, e=0, t=0$, and $r=5$. Since all associated weights were equal, the procedure sought the best compromise among all performance measures. $t=e=0$, meaning that no goal number of selected features was imposed, giving the procedure opportunities to exploit all available feature combinations to achieve the best performance. In this initial run, we obtained a feature subset of 18 in size.

For the subsequent runs, the value of $t$ was decreased starting from $17(=18-1)$ until performances were significantly worse than those of Hwang et al. $^{7}$ In order to obtain feature subsets of reduced sizes, parameters were set: $k=2, w_{P}=1.03, w_{R}=1$, $w_{F}=1, w_{M}=1, \rho=0.005, e=0.01$, and $r=5$. The reason for setting $w_{P}=1.03$ was to prevent the true positive rate from decreasing too much. In addition, $e>\rho$ was to allow the procedure to be proceeded to fewer features. The above settings were used to encourage selection of reduced feature subsets.

For each setting of $t$, we executed the procedure 10 times with different $k$-fold partitions and obtained 10 feature subsets of the same size. Since these 10 resultant feature subsets were slightly different, we performed another 5-fold cross-validation with these feature subsets and compared their performance scores. The one with the highest score were finally preserved as our feature subset.

In addition to the methods of Hwang et al or Gustafson et al, ${ }^{21}$ we also used $\mathrm{mRMR}^{13}$ and $\mathrm{CMIM}^{14}$ feature selection methods for comparison. Using mRMR as an example, the data used in our feature selection procedure were input into the mRMR program, which produced the ranking score of each feature. The feature with the least score was removed first and a subsequent 5-fold cross-validation with the preserved features is performed to calculate the composite score $C(S)$. That is, in the $i$ th iteration, the features with the lowest $i$ ranking scores were removed and $C(S)$ was calculated. The removal and cross-validation procedure was repeated until no feature was preserved. The entire process (including random choice of $50 \%$ data and feature removal) was executed 10 times and the feature subset of the same size with the highest score was recorded.

Table 4 shows the selected feature subsets of different sizes for $S$. cerevisiae data. The second column of the table lists all selected features. Each $\mathrm{N}_{i}$ in the first row represents the feature subset of size $i, 4 \leq$ $i \leq 18$, which was found using our backward feature selection procedure. For each feature subset $\mathrm{N}_{i}$, a bullet $(\bullet)$ mark below in the same column was used to indicate which feature was included. The most competent feature subsets selected by CMIM and 


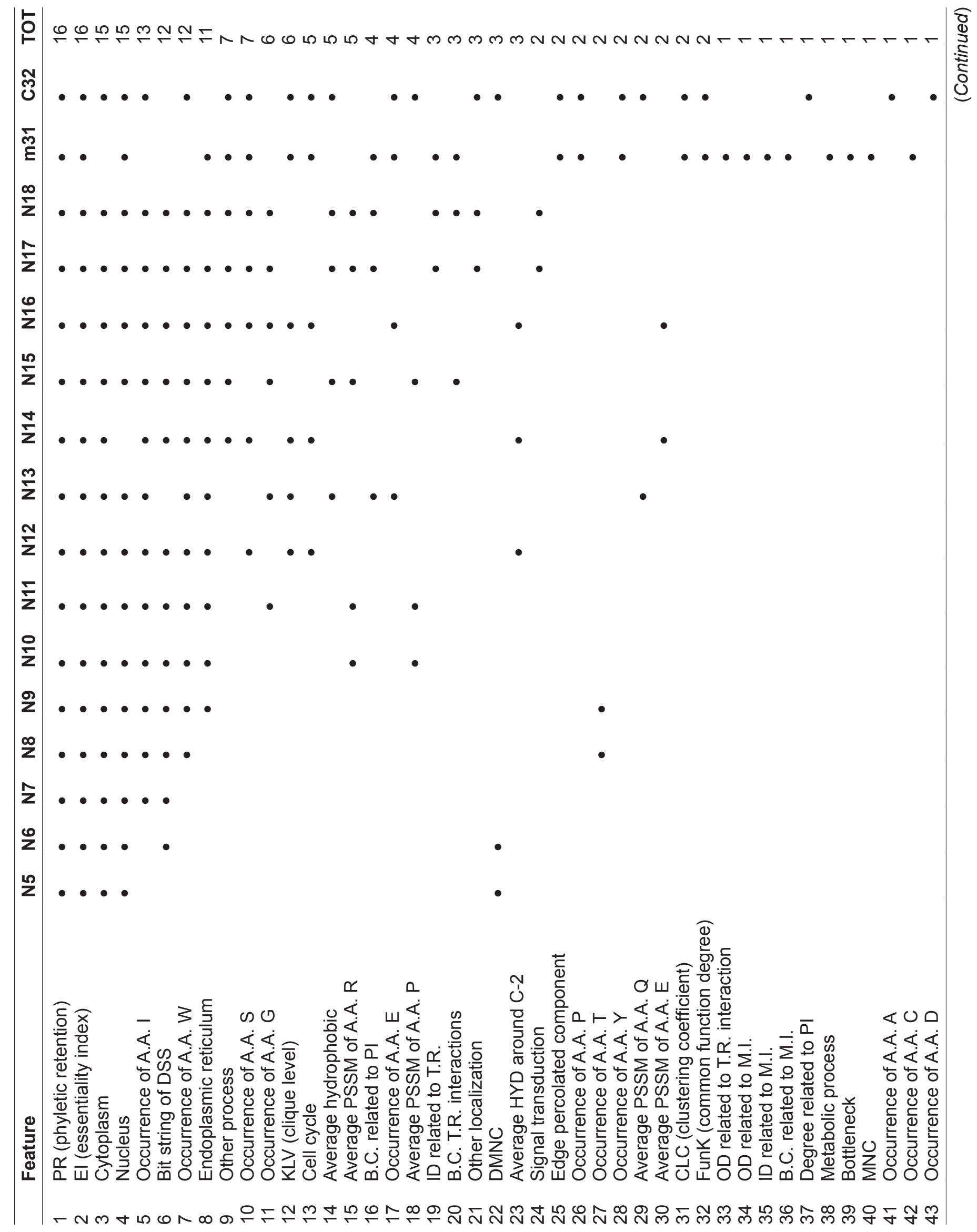




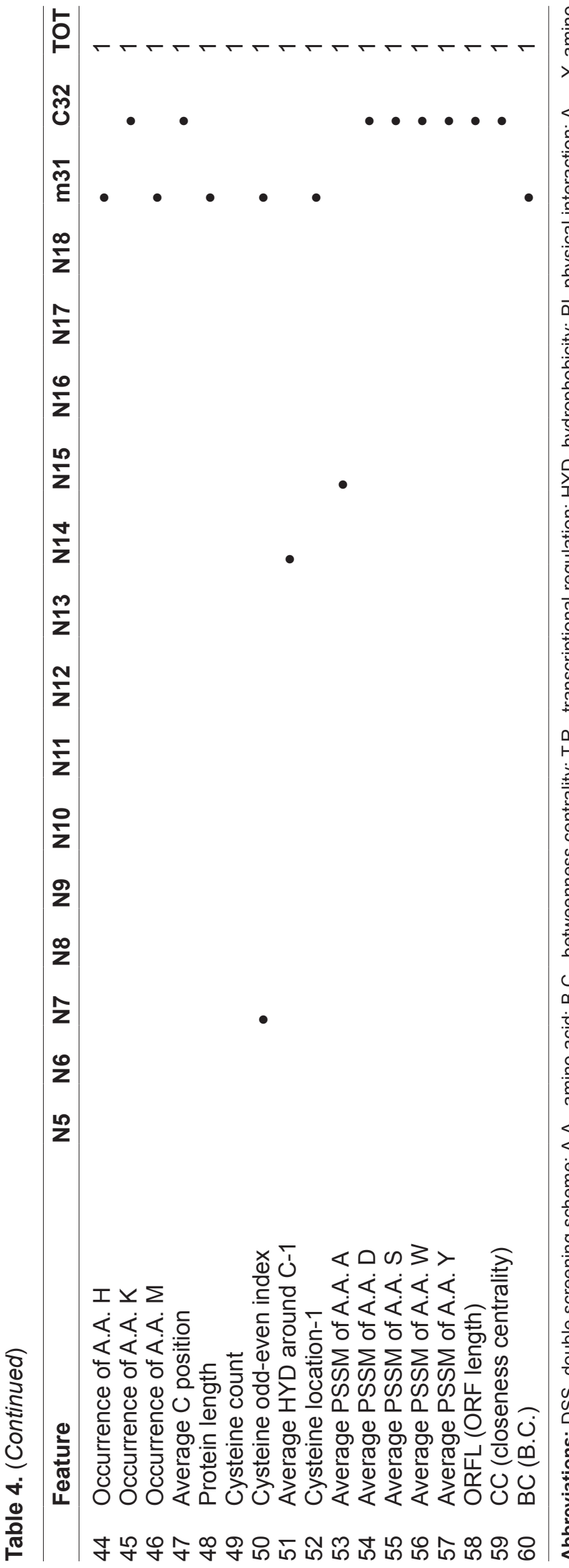

mRMR were denoted by $\mathrm{C} 32$ and $\mathrm{m} 31$, which means that 32 and 31 features were selected, respectively. It is observed that a total of 60 features have been selected, which represent the prominent proteins used to identify essential proteins.

After the feature subsets were selected, to conduct performance comparison as well as to cope with the randomness, we used Hwang's method to perform 1010 -fold cross-validations. Here, the true positive rates and false positive rates were input into a diferent software program to calculate ROC curves and AUC values. In this study, the software package we used is ROCR, which was developed by Tobias et al. ${ }^{30,31}$ Thus, the reported performance measures, including AUC, F1, MCC, precision, and recall values and ROC curves, were averaged over 10 10-fold crossvalidations.

For the $S$. cerevisiae data set, the predictor with Hwang's 10 features served as a benchmark because it yielded distinguished results in terms of feature size and performance. Additionally, mRMR or CMIM were adopted for comparison.

We appled the same procedure for the E. coli data set. The selected features are shown in Table 5, with a total of 43 features selected. In the table, feature subsets selected by CMIM and mRMR are denoted by $\mathrm{C} 9$ and $\mathrm{m} 13$, meaning that 9 and 13 features were selected, respectively.

\section{Bootstrap cross validations}

During the bootstrapping stage, for each bootstrap sample, an identical 10-fold partition was employed for all feature subsets to carry out cross-validations and compute various average performance measures. The procedure was repeated for 200 distinct bootstrap samples. In order to perform parametric significance tests, we evaluated whether the distribution of the resultant performance measures was normal and the variances obtained from different feature subsets were similar. Consequently, 200 results of each performance measure for each feature subset were subjected to the Kolmogorov-Smirno test. ${ }^{31}$ This test examines the null hypothesis that no systematic difference exists between the standard normal distribution and the underlying distribution against the alternative one that asserts a systematic difference. The threshold was set to 0.05 . If the $P$-value was less than 0.05 , we rejected the null hypothesis. For CMIM and 
Table 5. Selected features for $E$. coli data set.

\begin{tabular}{|c|c|c|c|c|c|c|c|c|c|c|c|c|c|c|}
\hline & Feature & N4 & N5 & N6 & N7 & N8 & N9 & N10 & N11 & N12 & N13 & C9 & m13 & TOT \\
\hline 1 & PR (phyletic retention) & - & • & - & - & - & - & - & - & - & - & - & - & 12 \\
\hline 2 & Open reading frame length & & - & & - & - & - & - & - & - & - & & & 8 \\
\hline 3 & Average PSSM of A.A. C & - & - & - & - & - & & & & & - & & & 6 \\
\hline 4 & Degree related to F.I. & - & - & - & - & - & & & & & - & & & 6 \\
\hline 5 & Degree related to A.I. & & & & & & - & - & - & - & & - & & 5 \\
\hline 6 & Degree related to $\mathrm{PI}$ & & & & & & - & - & - & • & & & - & 5 \\
\hline 7 & Average PSSM of A.A. A & & & & - & - & & & & & & - & - & 4 \\
\hline 8 & Average PSSM of A.A. R & & & & & & - & - & - & - & & & & 4 \\
\hline 9 & Average hydrophobic & & & & & & - & - & - & - & & & & 4 \\
\hline 10 & Bit string of DSS for PI & & & & & & - & - & - & - & & & & 4 \\
\hline 11 & Paralog count & & & - & & & & & & - & - & & - & 4 \\
\hline 12 & Occurrence of A.A. M & & & & & - & • & & & • & & & & 3 \\
\hline 13 & Occurrence of A.A. W & & & & & & & - & & & - & - & & 3 \\
\hline 14 & Occurrence of A.A. E & & - & - & & & & & & & & & & 2 \\
\hline 15 & Occurrence of A.A. F & & & & & & & & - & & & - & & 2 \\
\hline 16 & Occurrence of A.A. G & & & & & & - & & - & & & & & 2 \\
\hline 17 & Occurrence of A.A. I & & & & & & & & & & & - & - & 2 \\
\hline 18 & Average PSSM of A.A. Y & & & & & $\bullet$ & & & - & & & & & 2 \\
\hline 19 & Cysteine location-4 & & & & & & & & & & & - & - & 2 \\
\hline 20 & KLV (clique level) for PI & & & & - & & & & & & - & & & 2 \\
\hline 21 & Degree related to $\mathrm{PI}$ and $\mathrm{GC}$ & & & & & & & & - & • & & & & 2 \\
\hline 22 & Strand bias & & & & & & & & & - & & & - & 2 \\
\hline 23 & Occurrence of A.A. A & & & & & & & - & & & & & & 1 \\
\hline 24 & Occurrence of A.A. C & & & & & & & & & & & - & & 1 \\
\hline 25 & Occurrence of A.A. H & & & & & & & & & & & - & & 1 \\
\hline 26 & Occurrence of A.A.P & & & - & & & & & & & & & & 1 \\
\hline 27 & Occurrence of A.A. S & & & & & & & - & & & & & & 1 \\
\hline 28 & Average PSSM of A.A. N & & & & & & & & & & - & & & 1 \\
\hline 29 & Average PSSM of A.A. G & & & & & & & & & & - & & & 1 \\
\hline 30 & Average PSSM of A.A. K & & & & - & & & & & & & & & 1 \\
\hline 31 & Average PSSM of A.A. F & & & & & - & & & & & & & & 1 \\
\hline 32 & Average PSSM of A.A. T & & & & & & & & & & - & & & 1 \\
\hline 33 & Average PSSM of A.A. V & & & & & & & & & & - & & & 1 \\
\hline 34 & Average distance of every two Cs & & & & & & & & & - & & & & 1 \\
\hline 35 & Average HYD around C-2 & & & & & & & & & & - & & & 1 \\
\hline 36 & Cysteine location-1 & & & & & & & & & & & & - & 1 \\
\hline 37 & Cysteine location-5 & & & & & & & & & & & & - & 1 \\
\hline 38 & Cysteine odd-even index & & & & & & & & & & & & - & 1 \\
\hline 39 & Protein length & - & & & & & & & & & & & & 1 \\
\hline 40 & Bottleneck for PI & & & & & & & & & & & & - & 1 \\
\hline 41 & CC (closeness centrality) for PI & & & & & & & & & & & & - & 1 \\
\hline 42 & MNC for PI & & & & & & & & & & - & & & 1 \\
\hline 43 & B.C. related to all F.I. & & & & & & & & & & & & - & 1 \\
\hline
\end{tabular}

Abbreviations: C9, CMIM09; m13, mRMR13; TOT, total; DSS, double screening scheme; F.I., integrated functional interaction; A.I., all interactions. PI, physical interaction; HYD, hydrophobicity; A.A., amino acid; A ... Y, amino acid abbreviation.

mRMR, only the most prominent values are shown. Figures 2 and 3 illustrate the results for $S$. cerevisiae and $E$. coli data sets, respectively, in which the test values were recorded according to the feature subsets, performance measures, and experiment types. For the $S$. cerevisiae data set, the lowest $P$-value, 0.186 , was observed for AUC of the N8-imbalanced experiment. Therefore, it is likely that there was no significant difference between the normal distribution and the distribution of every performance measure of each feature subset. For the E. coli data set, most performance measures were normal with the exception of 

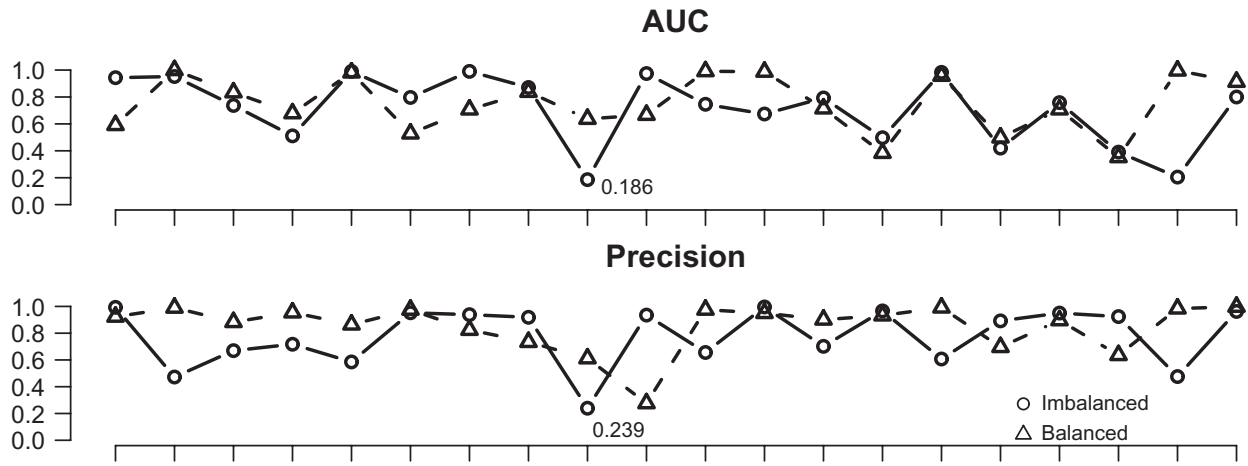

Recall

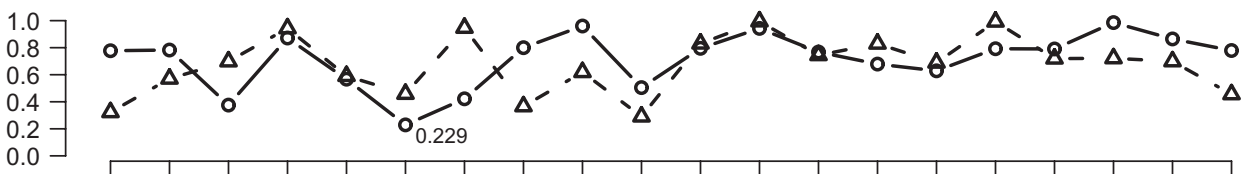

F1
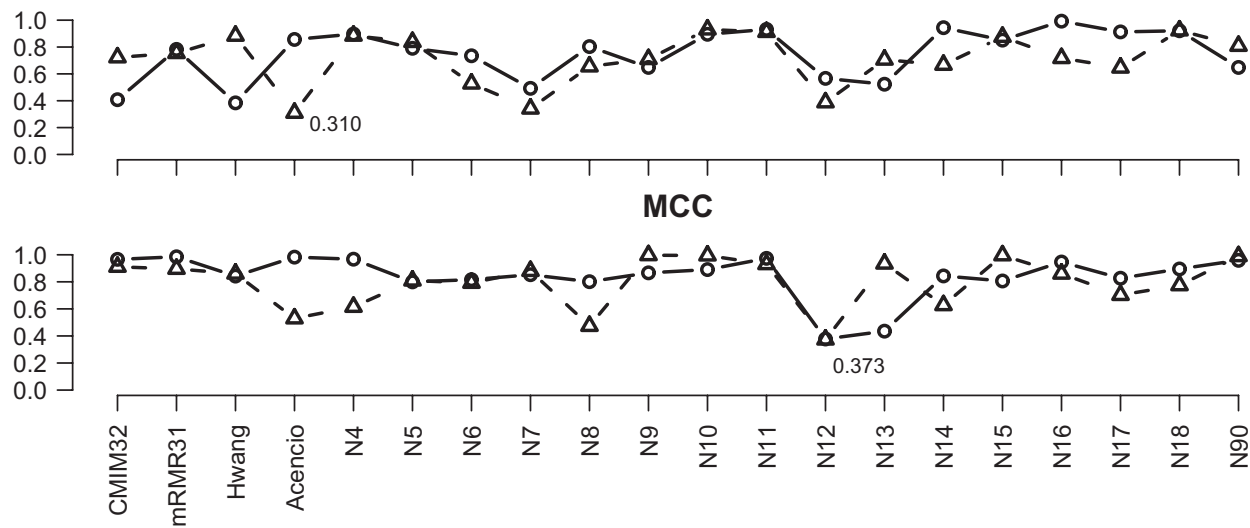

Figure 2. The $P$-value of the normality test in $S$. cerevisiae data set.

the recall values associated with N12-balanced (with $P$-value $=0.035)$, for which comparing results were not reliable. For the performance measures associated with each model, we also listed their confidence interval and information odds ratios, ${ }^{32}$ which are shown in the Appendix.

For a certain performance measure, since the variances obtained by various feature subsets were quite similar, we used an analysis of variance ${ }^{33}$ (ANOVA) test to examine whether differences existed among performance measures of different feature subsets. Here, one variance can be obtained from the multiple experiments with a feature subset. Differences existed according to the ANOVA. Next, all of these measures were compared with their associated benchmark to calculate performance deviations. The average deviation corresponding to each type of performance measure was evaluated using the $95 \%$ confidence interval covering 0 to determine significance.

\section{Performance comparison and significance tests}

In this section, we compared our experimental results with those associated with other feature selection methods and previous studies. For conciseness, we only show the most prominent results associated with mRMR and CMIM. We observed that feature sizes identified by these two methods were relatively large. To compare the feature subsets of smaller sizes, their comparison and their working principles are detailed in the Appendix.

\section{S. cerevisiae}

Table 6 lists the average values of five performance measures associated with a variety of feature subsets, which were obtained by 1010 -fold cross-validations for imbalanced data. We adjusted the SVM cost parameters in order to achieve similar levels of precision. The first four rows show results of CMIM32 (32 features), 

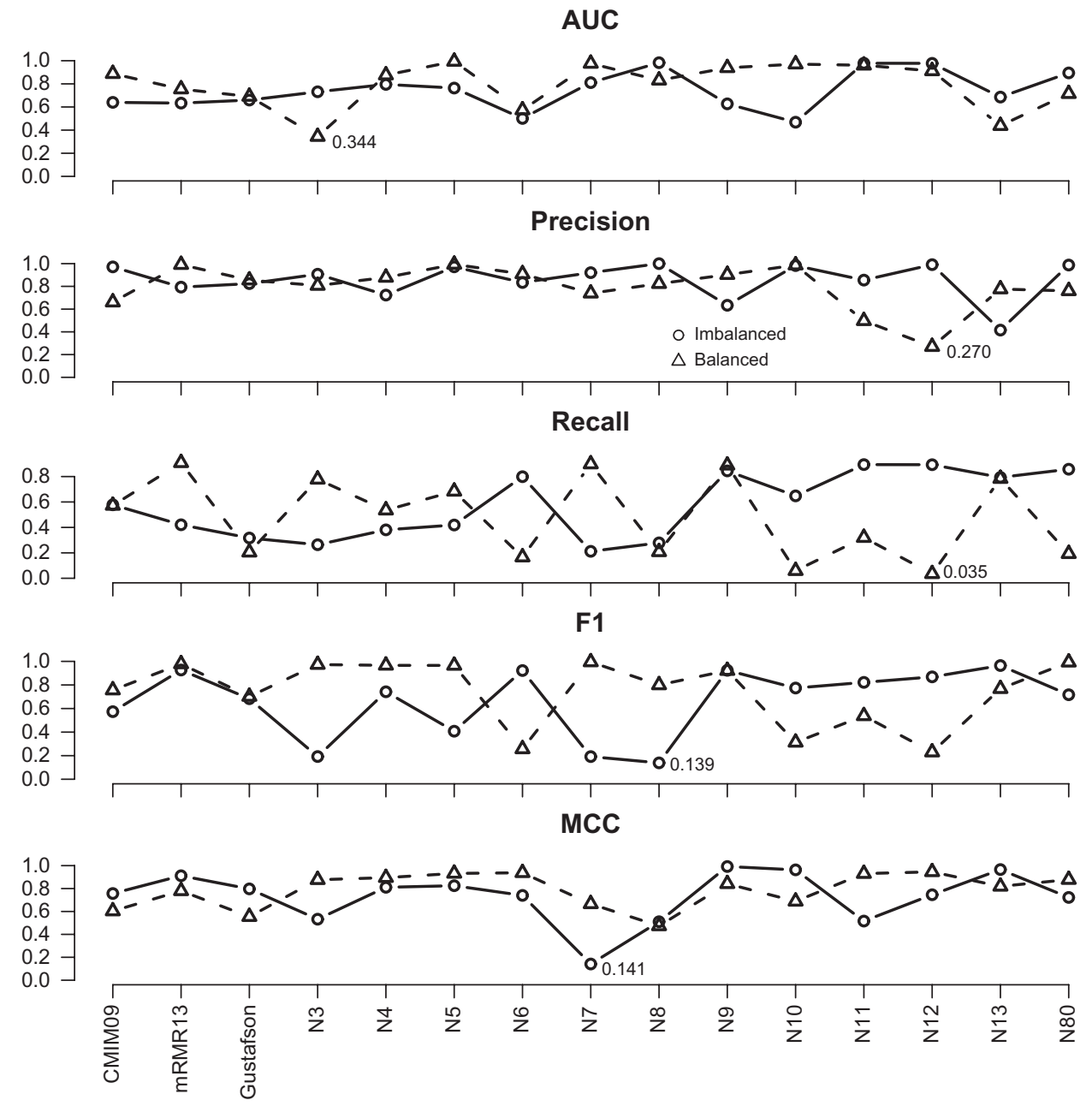

Figure 3. The $P$-value of the normality test in $S$. cerevisiae data set.

mRMR31 (31 features), Hwang's (10 features), and Acencio's (23 features). Values following these items are enclosed by parentheses and represent the numbers of features. Results produced by our method are listed in the subsequent rows of the table. Significance tests were carried out with the bootstrap cross-validations over 200 bootstrap samples. The first three symbols, which can be plus $(+)$ or minus $(-)$, following each numerical value represents significantly higher or lower than benchmark results. For those which serve as benchmarks are marked by star $\left(^{*}\right)$ symbols for clarity. For example, the recall of N6 was significantly higher than that of Hwang, while its AUC was significantly lower than those of mRMR31 and Hwang. For the feature subsets with a prefix name ' $\mathrm{N}$ ', their fourth symbols behind numerical values are used to indicate the significance between two neighboring rows. For example, for N7, its AUC was significantly higher than that of N6 and its recall value was also significantly higher than that of N8. For values of the same performance measure in each column, the best is underlined. Values in the last row show the results with the full set of 90 features.

Based on Table 6, CMIM32, mRMR31 and Hwang's predictors outperformed Acencio's in all performance measures. For our feature subsets, the performance measures were slightly higher than Hwang's. For those of N8, there was no performance difference from Hwang's in AUC, while the remaining measure values were higher than Hwang's. When the feature size exceeded 8 , except for precision values, improvement over Hwang's was consistently significant in most cases. For comparison with mRMR, our method performed nearly as well as mRMR3 1 when the feature size was between 9 and 13 with the exception of AUC values. When the feature size ranged from 14 to 18 , there was no performance difference between our model and mRMR31. 


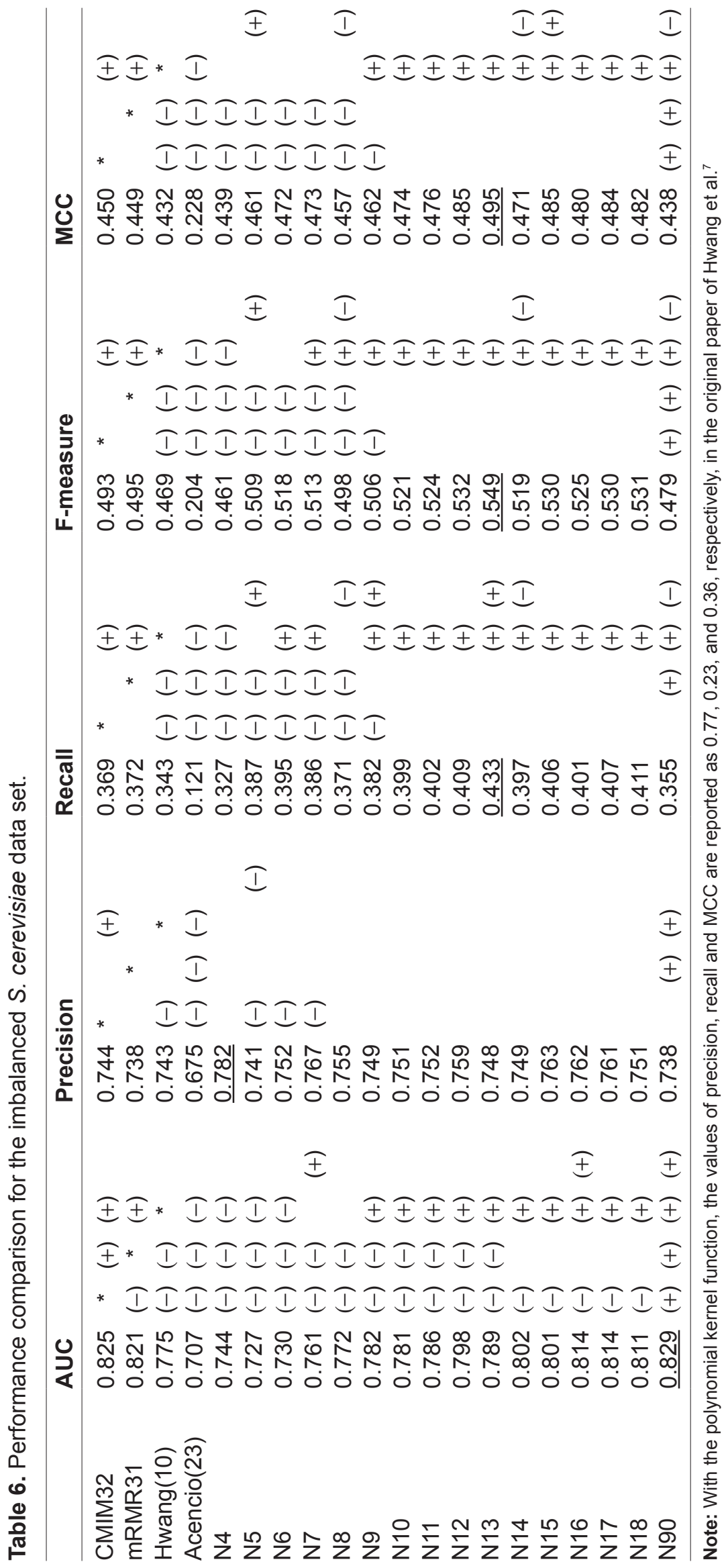


The most prominent predictor was CMIM32. Except for AUC values, our results achieved similar levels of performance when the size of features exceeded 14 . Note that the number of features in CMIM32 and mRMR31 were 32 and 31, which was much higher than ours.

Table 7 shows the average performance measures in balanced experiments of the $S$. cerevisiae data set, which were also obtained via 10 10-fold cross-validations. For those of our feature subsets with size ranging from 5 to 18 , nearly all performance measures were the same as or higher than those of Hwang's. This shows that the feature subsets with sizes exceeding 5 are at least as good as Hwang's. Additionally, those with 12 or more features achieved significant improvement. Compared with CMIM32 and mRMR31, our results showed similar levels of performance when the size of features exceeded 15 . The results with the full set of 90 features are shown in the last row, whose performance measures were similar to those from N14 to N18.

In Table 6, we can observe that feature subsets N5, N7, N9, N13, N15, and N16 showed significant improvement in performance but were smaller in feature sizes when compared with neighboring rows. In Table 7, the significant subsets were N5, N6, and N9. In addition, as shown in Tables 6 and 7, our models performed equally well as CMIM32 and mRMR31 when the feature size was 16 or 17 . We used N5, N9, and N16 to draw ROC curves.

\section{E. coli}

Tables 8 and 9 shows the average values of five performance measures associated with a variety of feature subsets, which were obtained by 1010 -fold cross-validations for imbalanced and balanced experiments, respectively. The first two rows show results of CMIM09 (9 features) and Gustafson's (29 features).

Table 8 shows that Gustafson's predictors outperformed CMIM09 in most performance measures in imbalanced experiments. For our feature subsets, the performance measures were slightly higher than CMIM09. When the feature size exceeded 6, the improvement over CMIM09 was consistently significant. To compare Gustafson's method with our method, ours almost performed as well as Gustafson's when the feature size was over 11. Note that the number of features in Gustafson's was 29, which was higher than ours. Table 9, except for the least effective predictor mRMR13, shows almost no performance difference among most feature subsets in balanced experiments. For further ROC analysis, in addition to CMIM09, mRMR13, and Gustafson's, we further used N4, N8, N11 and N80 to draw ROC curves. This is because we observed performances of insufficient, middle and full feature sets.

\section{ROC analysis \\ S. cerevisiae}

Figure 4 illustrates the average ROC curves and AUCs of various feature subsets for the imbalanced data experiments. Apart from the most competent predictor CMIM32, although the AUC of N5 is higher than that of Acencio's, an intersection can be observed at 0.5 on the horizontal axis. This indicates that N5 was a better predictor when the allowed maximal false positive rate was below 0.5 . In contrast, when the allowed false positive rate exceeded 0.5, Acencio's was better than N5. Comparing N9 and Hwang's method, both AUC values were similar. For the feature subsets with sizes exceeding 8 (not all shown in this figure), all true positive rates were either higher or at least close to Hwang's. This was also supported by the significance tests in Table 6 and suggests that the feature subsets with sizes exceeding 8 achieved higher performance in AUC than Hwang's predictor.

Figure 5 illustrates the average ROC curves and AUCs of various feature subsets for the balanced data experiments. CMIM32 again was the most competent predictor. Additionally, N16 also achieved the same level of AUC. For the feature subsets of sizes ranging from 5 to 18 (not all shown), their true positive rates were either higher or at least close to Hwang's level. Thus, N5, N6, .., N18 outperformed or performed equally well for various combinations of true and false positive rates in the balanced experiments. Similarly to the imbalanced data set, the more features, the higher the AUC values. However, the improvement in AUC over the feature addition was not as significant as those in the imbalanced experiments. It should be noted that both the ROC curve and AUC of Acencio's predictor were reproduced by our experiments and thus they were slightly different from the original values reported by Acencio and Lemke. ${ }^{5}$ 


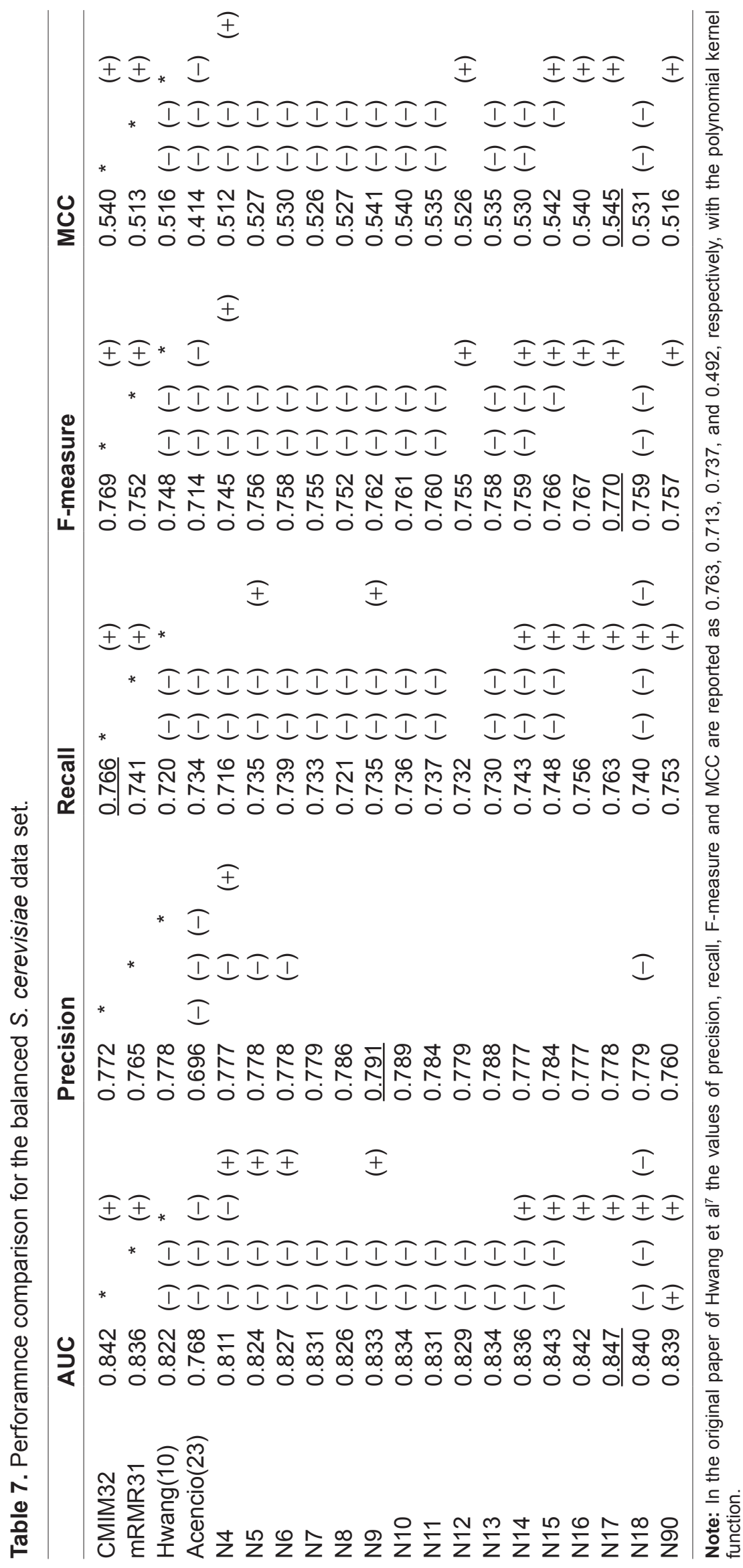




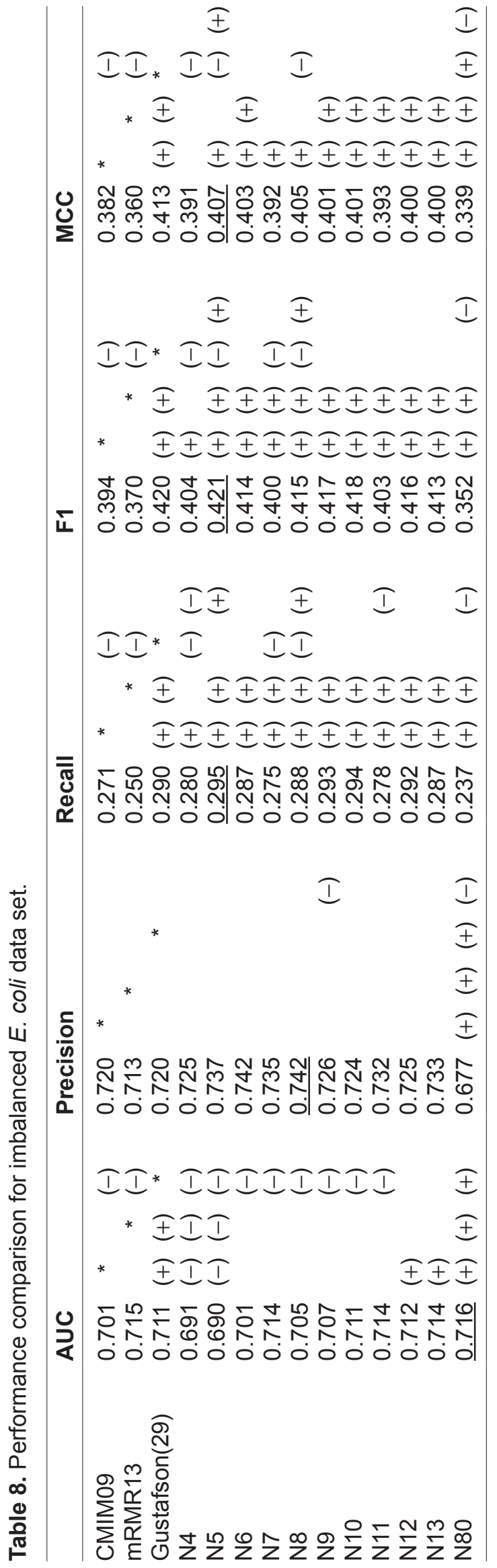

E. coli

For imbalanced data set, Figure 6 illustrates the average ROC curves and AUCs of various feature subsets. It shows that all curves were similar below the $10 \%$ horizontal range. This indicates that there was little difference when the allowable false positive rate was less than $10 \%$. For the horizontal range above $10 \%$, N80 was the highest performer, Gustafson and N11 were secondary, and N4 was the worst. In contrast to the imbalanced data set, for Figure 7 corresponding to the balanced data set, N4 and N8 were the best performers. The remaining predictors showed few differences.

\section{Top percentage analysis}

S. cerevisiae

Table 10 shows the average top percentage information for the imbalanced data set. The top $\theta$ probability is defined as the ratio of the number of truly predicted essential proteins over the top-ranked $\theta \times$ 975 proteins, where the total number of true essential proteins is 975 . The top $\theta$ probability shows the likelihood that the proteins are essential if the user decides to choose a specific number of top-ranked candidates. It is slightly different from precision because the top-ranked candidates (or denominator) are not necessary to be classified as essential. CMIM32, mRMR31 and Hwang's results again served as benchmarks and they are denoted by star '*' symbols in the table. The minus symbol following each value represents that the value was lower than the benchmark results.

Both mRMR31 and Hwang's predictor were extremely effective within the $10 \%$ range. This indicates that these predictors were quite preferable when the total number of true essential proteins was known and the allowable top-ranked candidates were within $10 \%$. Most of our predictors outperformed them beyond $10 \%$. For CMIM32, our predictors outperformed it beyond $30 \%$. Thus, N14 may be a better choice because it is relatively effective beyond $10 \%$. Figure 8 depicts the average top percentage curves.

E. coli

Table 11 shows the average top percentage information for the imbalanced data set. CMIM09, mRMR13, and Gustafson's results serve as benchmarks. 
Table 9. Performance comparison for balanced E. coli data set.

\begin{tabular}{|c|c|c|c|c|c|}
\hline & AUC & Precision & Recall & F1 & MCC \\
\hline CMIM09 & $0.767 * \quad(+)$ & 0.720 * & $0.700 * \quad(+)$ & $0.710 *(+)$ & $0.421 *$ \\
\hline mRMR13 & $0.762(-){ }^{*}(-)$ & 0.728 & $0.654(-) *(-)$ & $0.689(-) *(-)$ & $0.396 *(-)$ \\
\hline Gustafson(29) & $0.777 \quad(+)$ * & 0.722 & $\underline{0.715} \quad(+) *$ & $0.719 \quad(+)$ * & $0.440(+) *$ \\
\hline N4 & 0.780 & 0.733 & $\overline{0.701}$ & $\overline{0.717}$ & $0.446(+)$ \\
\hline N5 & 0.779 & 0.730 & 0.706 & 0.718 & $0.445(+)$ \\
\hline N6 & $(-)(-)$ & 0.735 & $(-)(-)$ & 0.696 & 0.425 \\
\hline N7 & $(+) \quad(+)$ & $\underline{0.737}$ & 0.696 & 0.716 & $\underline{0.448}(+)$ \\
\hline N8 & $\overline{0.781}$ & $\overline{0.723}$ & 0.711 & 0.717 & $\overline{0.439}(+)$ \\
\hline N9 & 0.782 & 0.715 & 0.703 & 0.709 & 0.423 \\
\hline N10 & 0.781 & 0.725 & 0.702 & 0.713 & $0.436(+)$ \\
\hline N11 & 0.777 & 0.719 & 0.700 & 0.709 & 0.426 \\
\hline N12 & 0.776 & 0.715 & 0.695 & 0.705 & 0.418 \\
\hline N13 & 0.776 & 0.731 & 0.695 & 0.712 & $0.439(+)$ \\
\hline N80 & 0.769 & 0.711 & 0.715 & 0.713 & 0.424 \\
\hline
\end{tabular}

The CMIM09 predictor was the most effective over the entire range. Most of our predictors outperformed these predictors beyond $15 \%$. N9 was the most prominent since it was relatively effective over the entire range. Figure 9 depicts the average top percentage curves.

\section{Discussion}

By inspecting the $S$. cerevisiae feature subsets listed in Table 4, we observed that the most prominent features indeed come from diverse sources. This includes sequence, protein, topology and other properties. Among these features, amino acid occurrence I,

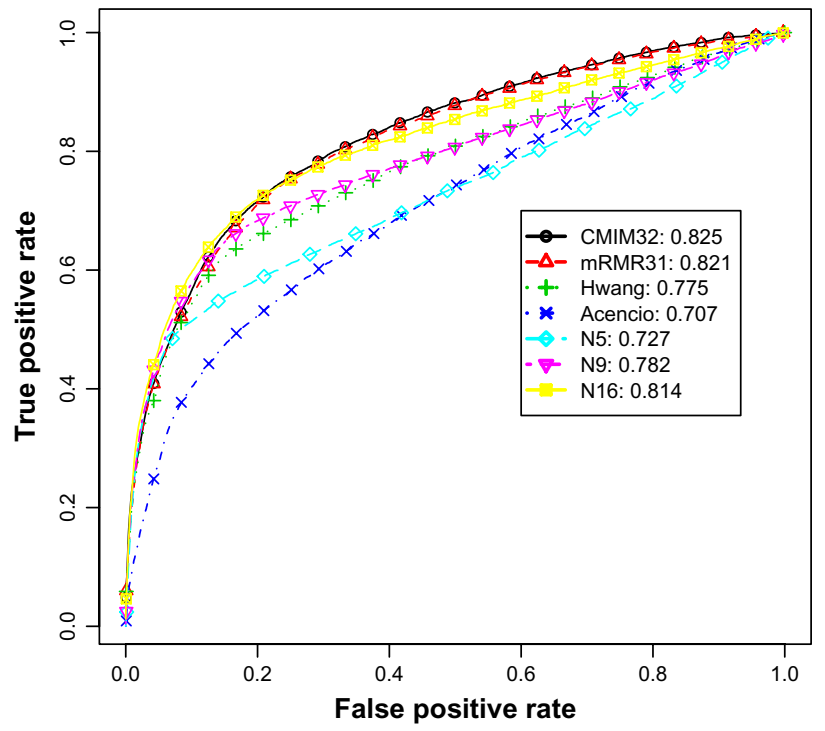

Figure 4. The average ROC curves and AUCs for the imbalanced S. cerevisiae data set. amino acid occurrence $\mathrm{W}$, bit string of double screening scheme, cytoplasm, endoplasmic reticulum, EI (essentiality index), nucleus, and PR (phyletic retention) were selected more than 10 times. Two among the above features, EI and PR, were included in all feature subsets and thus they are regarded as the most important factors for identifying essential proteins. N9 and N8 were the feature subsets that cover most of the above 8 features. Their prediction capability associated with these two feature subsets outperformed Hwang's results in all performance measures, except for AUC and the top percentage probability at a very low value. Furthermore, two amino acids, which

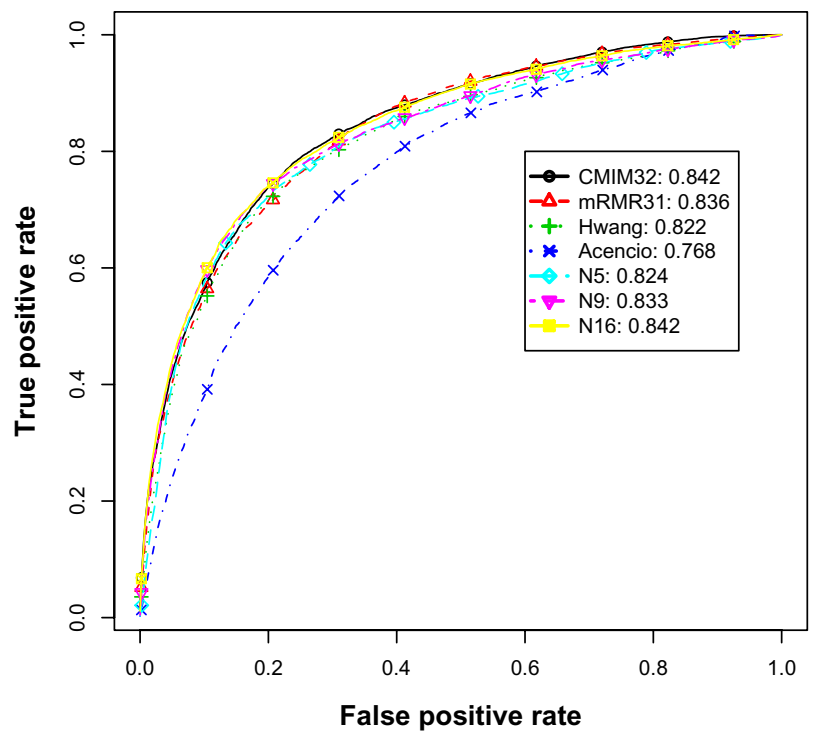

Figure 5. The average ROC curves and AUCs for the balanced S. cerevisiae data set. 


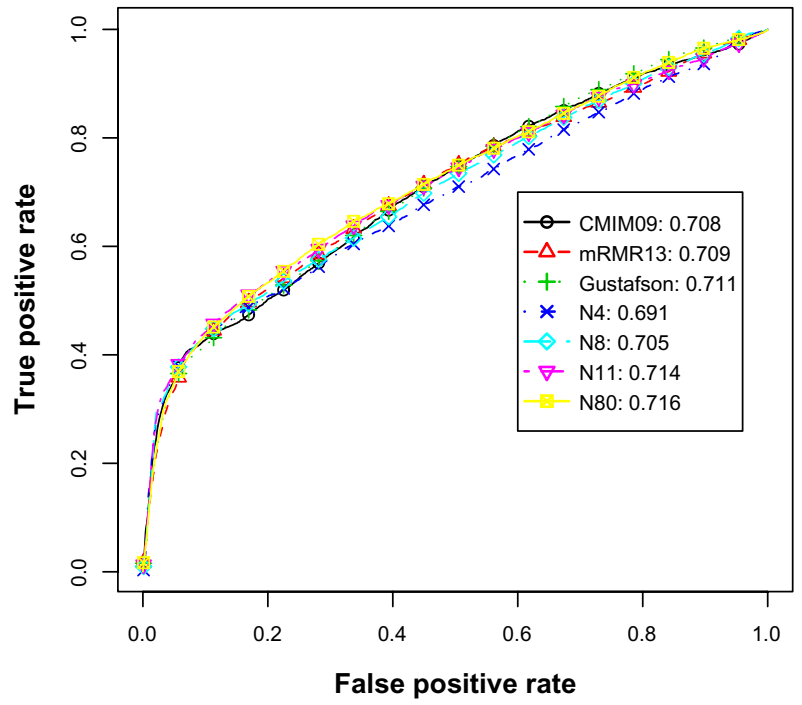

Figure 6. The average ROC curves and AUCs for the imbalanced E. coli data set.

were relatively easy to extract, were included in these two feature subsets. For predictors that were built by the feature subsets of 10 or more features, they were consistently superior to Hwang's in nearly all performance measures. Interestingly, mRMR and CMIM selected several sequence-derived features, such as PSSM and amino acid occurrence. It thus seems these features are good for essentiality prediction in terms of relevance and feature independence. By analyzing Tables 6 and 7, we recommend using N16, N9, and N6. N16 performed nearly as well as CMIM32 (or mRMR31) and was more compact in feature size. For

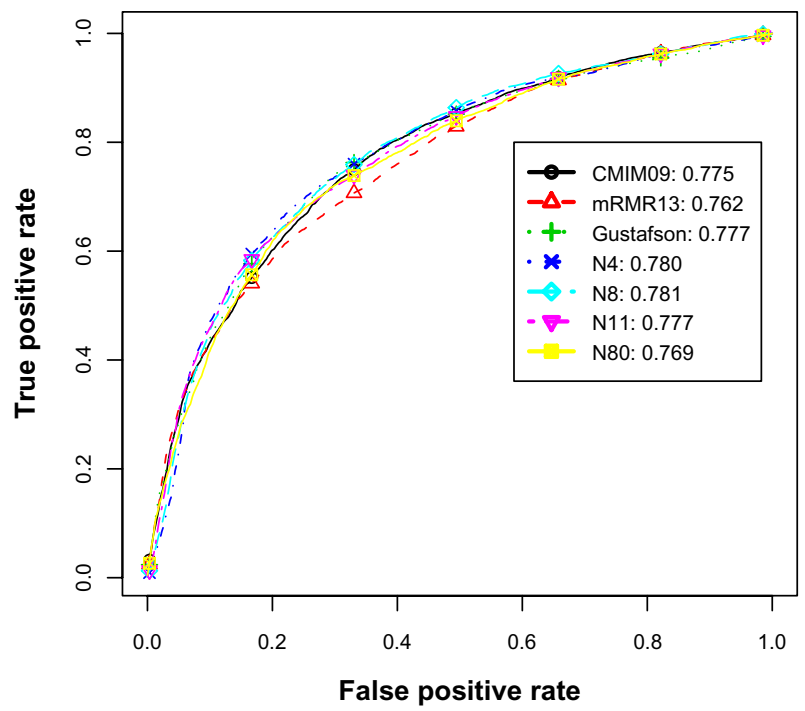

Figure 7. The average ROC curves and AUCs for the balanced E. coli data set.
N9 and N6, by choosing one additional feature, they were significant higher than N8 and N5 in some performance measures.

For the E. coli features listed in Table 5, the most and second important features were PR (phyletic retention) and open reading frame length. The rest of important features which were selected more than five times included: average PSSM of amino acid $\mathrm{C}$ and degree related to integrated functional interactions. N5, N7, N8, and N13 covered most of these features. Among the 43 listed features, 21 sequence-related features, such as amino acid occurrence and average PSSM, were selected. In this data set, we recommend feature subsets of sizes exceeding 11 because of their effectiveness and compactness in feature size.

With experimental results for the two data sets, we conclude that phyletic retention is the most important feature for identifying essential proteins. It is defined as the number of present ortholog organisms. Gustafson et al study ${ }^{21}$ analyzed different organisms to calculate phyletic retention for $E$. coli and $S$. cerevisiae data sets. This is sensible because different species may be associated with different organisms. From the biological view, the retention process over long evolutionary periods suggests that some organisms are crucial for certain cell functionality. By inspecting the top 5 occurrences of amino acids in Tables 4 and 5, we find Tryptophan (W) and Glycine (G) were two top-ranking features. Since both these two amino acids are non-polar and hydrophobic, we may hypothesize that either essentiality is related to these physicochemical properties or that the features possessing discrimination information is not captured by other top-ranking features.

In this study, we compiled various interaction information including physical, metabolic, transcriptional regulation, and integrated functional and genomic context interactions. The experimental results revealed that various properties, such as degrees, were more or less identified as important features. This implies that the interaction information, not limited to physical interactions, may also be closely related to essential properties. According to the literature, hubs of the networks, possessing abundance of interaction partners, are important due to the fact that they play central roles in mediating interactions among numerous 
Table 10. Percentage of essential proteins in the imbalanced S. cerevisiae data.

\begin{tabular}{|c|c|c|c|c|c|c|c|c|c|}
\hline & Top 5\% & Top 10\% & Top 15\% & Top 20\% & Top 25\% & Top $30 \%$ & Top 50\% & Top 75\% & Top $100 \%$ \\
\hline CMIM32 & $0.939 *--$ & $0.918^{*}$ & $0.910^{*}$ & $0.892^{*}$ & $\underline{0.870^{*}}$ & $0.839^{*}$ & $0.743^{*}$ & $0.645^{*}$ & $0.582^{*}$ \\
\hline mRMR31 & $0.955 *-$ & $0.905-{ }^{*}-$ & $0.884-^{*}$ & $0.862-^{*}$ & $\overline{0.834}{ }^{*}-$ & $0.820-^{*}$ & $0.740-^{*}$ & 0.641 -* $^{*}$ & $0.572-^{*}$ \\
\hline Hwang(10) & 0.959 & $0.918 *$ & $0.871--^{*}$ & $0.853--^{*}$ & $0.843-*$ & $0.816--^{*}$ & $0.720--^{*}$ & $0.637--^{*}$ & $0.563--^{*}$ \\
\hline Acencio(23) & $0.800---$ & $0.741---$ & $0.693---$ & $0.661---$ & $0.646---$ & $0.625---$ & $0.578---$ & $0.519---$ & $0.457---$ \\
\hline N4 & $\underline{0.980}$ & 0.930 & $0.905-$ & $0.877-$ & $0.865-$ & 0.850 & $0.727--$ & $0.632---$ & $0.559---$ \\
\hline N5 & $0.843---$ & $0.861---$ & $0.859---$ & $0.852---$ & $0.841-$ & $0.827-$ & 0.751 & $0.641-$ & $0.530---$ \\
\hline N6 & $0.908---$ & $0.894---$ & $0.875--$ & $0.857--$ & $0.850-$ & 0.834 & 0.763 & $0.635---$ & $0.526---$ \\
\hline N7 & $0.861---$ & $0.892---$ & $0.897-$ & $0.885-$ & $0.854-$ & $0.832-$ & 0.770 & $0.645-$ & $0.570--$ \\
\hline N8 & $0.892---$ & $0.904---$ & $0.895-$ & $0.877-$ & $0.868-$ & 0.850 & 0.751 & 0.657 & $0.574-$ \\
\hline N9 & $0.880---$ & $0.911-\quad-$ & $0.895-$ & $0.875-$ & $0.860-$ & 0.832 & 0.753 & 0.665 & 0.585 \\
\hline N10 & $0.882---$ & $0.896---$ & 0.893 & $0.882-$ & $0.858-$ & 0.846 & 0.762 & 0.665 & $0.581-$ \\
\hline N11 & $0.900---$ & $0.900---$ & $0.888-$ & $0.875-$ & $0.861-$ & $\underline{0.856}$ & 0.769 & 0.667 & $0.580-$ \\
\hline N12 & $0.941--$ & 0.924 & 0.899 & $0.872-$ & $0.866-$ & 0.854 & 0.776 & 0.664 & 0.588 \\
\hline N13 & $0.941--$ & 0.910 & $0.886-$ & 0.870 & $0.853-$ & 0.840 & $\underline{0.781}$ & 0.672 & $0.578-$ \\
\hline N14 & $0.949--$ & $\underline{0.932}$ & $\underline{0.916}$ & $\underline{0.897}$ & $0.867-$ & 0.845 & 0.759 & 0.667 & 0.587 \\
\hline N15 & $0.906---$ & $\overline{0.894}---$ & $\overline{0.897}-$ & $\overline{0.884}-$ & $0.866-$ & 0.851 & 0.776 & 0.672 & 0.584 \\
\hline N16 & $0.933---$ & $0.901---$ & $0.895-$ & $0.886-$ & $0.864-$ & 0.851 & 0.771 & 0.677 & $\underline{0.599}$ \\
\hline N17 & $0.943--$ & $0.903---$ & $0.879--$ & $0.871-$ & $0.866-$ & 0.856 & 0.777 & 0.679 & $\overline{0.595}$ \\
\hline N18 & $0.937---$ & $0.892---$ & $0.880--$ & $0.870-$ & $0.864-$ & 0.854 & 0.778 & $\underline{0.683}$ & 0.595 \\
\hline N90 & $0.939--$ & $0.911-\quad-$ & $0.884-$ & 0.869 & $0.856-$ & 0.835 & $0.728--$ & $\overline{0.639}--$ & 0.572 \\
\hline
\end{tabular}

less-connected proteins. Thus, proteins involved in the complex mediation processes are more likely to be crucial for cellular activity or survival.

For the feature selection proposed in this study, let the size of all available and target selected features be $m$ and $t$, and the maximal retry times be $r$. The number of SVM cross-validation times is between $1 / 2$ $(m+t+1) \times(m-t)$ and $1 / 2(m+t+1) \times(m-t) \times r$.

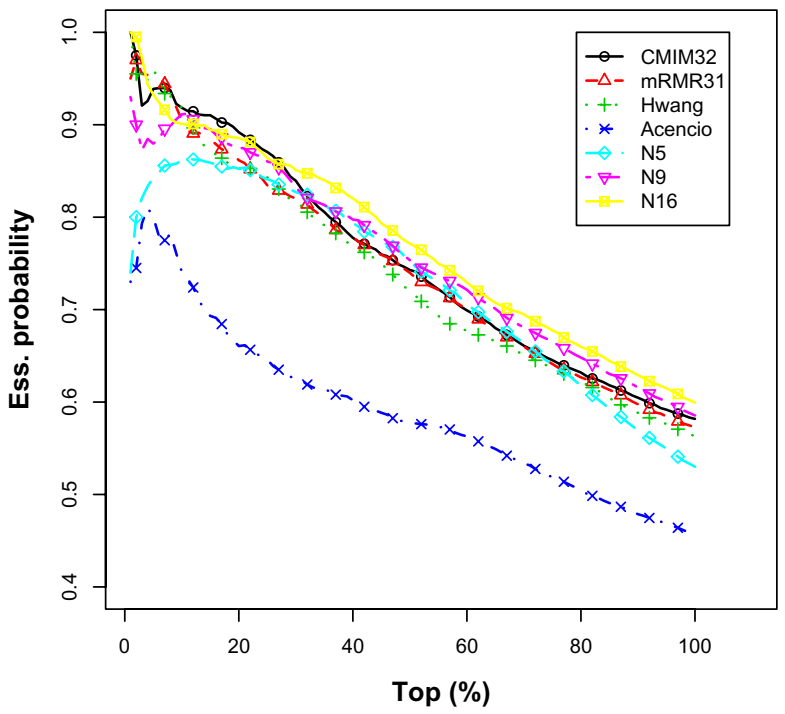

Figure 8. The average top percentage curves for the imbalanced S. cerevisiae data set.
It takes approximately 1 minute for the LIBSVM software to perform a 2-fold cross-validation on one Power5+ processor of IBM P595 computer. Assuming $m=90, t=10$ and $r=5$, the total running time is between 4,000 and 20,000 minutes. The IBM P595 allows users to manually submit several processes into the computer in order to speed up the execution. For example, we can invoke at most $10 \mathrm{SVM}$ processes simultaneously. Consequently, a maximal 10-time speed-up can be achieved and the total running time can thus be reduced.

If we inspect Tables 4 and 5, we can find that more than one-third of the features were not significantly relevant and thus were not selected. These features are relatively easy to remove during backward feature selection procedure at the beginning stage. According to the authors' experience, the rounds of retry $r$ are not critical in this stage. With an increasing number of features removed, the required number of retry must be increased as identifying relatively less competent features becomes increasingly difficult. The number of retry $r$ accompanied by the rest of user-specified parameters (such as the minimal improvement $\rho$ et al) was set appropriately to ensure that the feature selection procedure could proceed. 
Table 11. Percentage of essential proteins in the imbalanced E. coli experiment.

\begin{tabular}{|c|c|c|c|c|c|c|c|c|c|}
\hline & Top 5\% & Top 10\% & Top 15\% & Top $20 \%$ & Top 25\% & Top 30\% & Top 50\% & Top 75\% & Top $100 \%$ \\
\hline CMIM09 & $0.745^{*}$ & $0.775^{*}$ & $0.730^{*}$ & $0.730^{*}$ & $0.737^{*}$ & $0.727^{*}$ & $0.644^{*}$ & $0.542^{*}$ & $0.440^{*}-$ \\
\hline mRMR13 & $0.719-^{*}$ & $0.725-^{*}$ & $0.714-^{*}$ & $0.701-^{*}-$ & $0.690-^{*}-$ & $0.679-^{*}-$ & $0.614-^{*}-$ & $0.531-^{*}$ & 0.446 * \\
\hline Gustafson(29) & $0.706--^{*}$ & $0.692--^{*}$ & $0.705--^{*}$ & $0.707-$ * & $0.695-$ * & $0.685-*$ & $0.624-*$ & $0.522--^{*}$ & $0.436--^{*}$ \\
\hline N4 & $0.610---$ & $0.689---$ & 0.765 & 0.760 & $\underline{0.749}$ & 0.752 & 0.649 & $0.534-$ & 0.449 \\
\hline N5 & $0.655---$ & $0.705--$ & 0.747 & 0.747 & 0.745 & 0.743 & 0.653 & 0.535 & 0.443 \\
\hline N6 & $0.719-$ & $0.723--$ & $0.717-$ & 0.736 & 0.744 & 0.748 & 0.658 & $0.525--$ & $0.435---$ \\
\hline N7 & $0.568---$ & $0.700--$ & $0.713--$ & 0.730 & 0.748 & $\underline{0.762}$ & 0.655 & $0.540-$ & $\underline{0.464}$ \\
\hline N8 & $0.671---$ & $0.703--$ & $0.723-$ & 0.736 & $0.728-$ & 0.731 & 0.652 & 0.535 & 0.449 \\
\hline N9 & $\underline{0.813}$ & $\underline{0.785}$ & $\underline{0.766}$ & 0.754 & $0.734-$ & $0.726-$ & $\underline{0.685}$ & 0.548 & 0.459 \\
\hline N10 & 0.794 & $0.751-$ & $0.728-$ & 0.732 & 0.741 & 0.745 & 0.668 & $\underline{0.550}$ & 0.463 \\
\hline N11 & 0.719 & $0.721--$ & 0.734 & 0.742 & 0.748 & 0.748 & 0.668 & $\overline{0.539}$ & 0.457 \\
\hline N12 & $0.735-$ & $0.738-$ & 0.745 & 0.750 & 0.743 & 0.740 & 0.667 & 0.548 & 0.458 \\
\hline N13 & $0.655---$ & $0.672---$ & $0.713--$ & 0.739 & $0.736-$ & 0.732 & 0.668 & 0.548 & 0.457 \\
\hline N80 & $0.674---$ & $0.690---$ & $0.703---$ & $0.705--$ & $0.703-$ & $0.691-$ & $0.632-$ & $0.529--$ & 0.452 \\
\hline
\end{tabular}

\section{Conclusion and Future Work}

In this study, we incorporated several protein properties, including sequence, protein, topology, and other properties. There was a total of 55 groups and 96 features. The features were included in two data sets for experiments: $S$. cerevisiae and E. coli. We used a modified sequential backward feature selection to identify good feature subsets and used the SVM software tools for classifying essential proteins. In addition, we built several SVM models for both imbalanced and balanced data sets. As our experimental results illustrate, some features were indeed shown to be effective for essentiality prediction. Feature subsets selected by

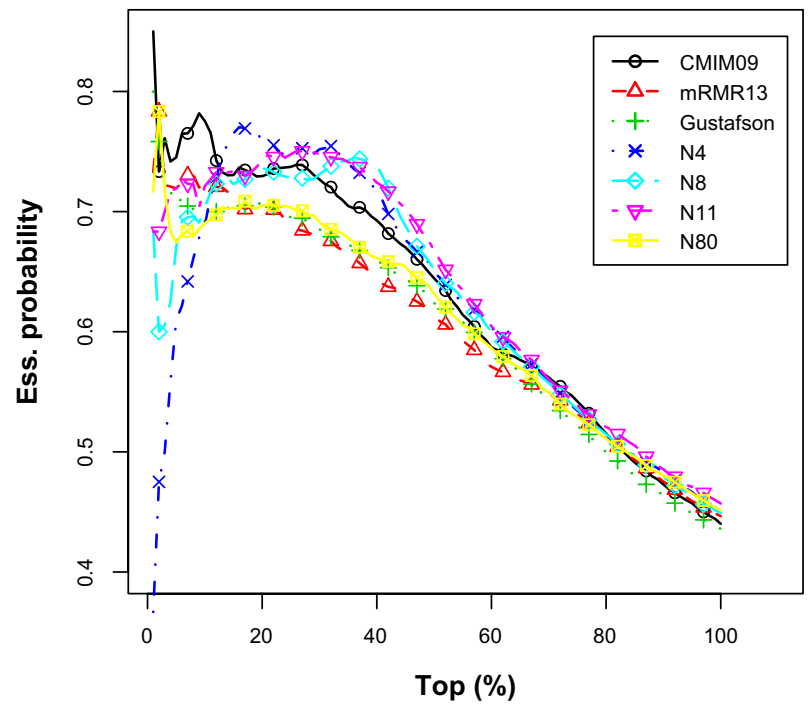

Figure 9. The average top percentage curves for the imbalanced E. coli data set. our method were effective in term of feature size and performance. This is because our method took both feature size and performance into consideration and consequently the resultant feature subset was considerably compact. We compared our experimental results by carrying out significance tests for several types of performance measures. Hence, this provides the potential researcher of essential proteins a practical guide to which feature or method is more prominent.

In the imbalanced $S$. cerevisiae data experiment, our best results for F-measure and MCC were 0.549 and 0.495 , respectively, which was associated with the N13 predictor. In contrast, for the same performance measures, we achieved 0.77 and 0.545 in the balanced data experiment, which were associated with the N17 predictor. The experimental results showed that the performance of our models was better than Hwang's when we selected more than 9 features. If achieving higher accuracy is the main issue, we recommend the N16 model (16 features). When one prefers a compact feature set of small size, we suggest using the N9 model (9 features). We also list important features. These features may be crucial for identifying essential proteins.

For $E$. coli data set, our best values of F-measure and MCC were 0.421 and 0.407 in the imbalanced experiments. In the balanced experiment, the best values of F-measure and MCC were 0.718 and 0.448 , respectively. Both of the best results were associated with the N5 predictor. For the data set, we found that predictors associated with the feature size above 11 were indeed comparable to Gustafsons'. 
There several possible methods for further improving the prediction capability. Features related to the protein sequence properties may also be useful for identifying essentiality. Furthermore, since proteins with similar primary structures may possess similar functions, thus the essentiality may be addressed from the sequence motif perspective. ${ }^{34}$ In addition to the above approaches, performance can be improved by incorporating other tools or constructing hybrid predictors. Among these, the majority vote ${ }^{35}$ is a strategy for combining classifiers. This method represents the simplest method for categorical data fusion. According to the literature, ${ }^{36}$ the prerequisite for improvement arises from the fact that each individual classifier must contain distinct information for discrimination. Otherwise, some negative effects may be imposed on the constructed ensemble.

\section{Appendix}

\section{Feature extraction}

a. Outdegree and indegree related to transcriptional regulation interaction: The feature represents the number of outgoing (or incoming) links to the gene $g$ corresponding to a protein. Links are represented in terms of transcriptional regulation interactions.

b. Betweenness centrality transcriptional regulation interactions: Let $\sigma_{g i g j}$ denote the number of shortest paths between $g_{i}$ and $g_{j}$. The value $\sigma_{g i g}(g)$ is defined as the number of shortest paths between $g_{i}$ and $g_{j}$ passing through $g$. Paths are represented in terms of transcriptional regulation interactions.

c. Betweenness centrality related to physical interactions: The value $\tau_{g i g j}(g)$ is defined as the number of shortest paths between $g_{i}$ and $g_{j}$ passing through $g$. The definition is similar to the previous one. However, the paths here are represented in terms of protein physical interactions.

d. Protein properties: Acencio and Lemke ${ }^{5}$ discovered that the integration of topological properties, cellular components, and biological processes possess good capability for predicting essential proteins. Hence, our features also contained cellular components (cytoplasm, endoplasmic reticulum, mitochondrion, nucleus or other localization) and biological processes (cell cycle, metabolic process, signal transduction, transcription, transport or other process).

The above four feature sets were obtained from Acencio and Lemke. ${ }^{5}$

e. Betweenness centrality related to integrated functional, PI and GC network: The values are defined identically as those mentioned above while the paths here are represented in terms of integrated functional, PI and GC network interactions.

f. Degree related to integrated functional, PI and GC network: The values were defined identically to those mentioned above, while the paths here are represented in terms of integrated functional, PI, and GC network interactions.

For the above two feature sets, we first collected network information from $\mathrm{Hu}$ et $\mathrm{al}^{22}$ and then conducted calculations using iGraph software. ${ }^{37}$

g. Maximum neighborhood component and density of maximum neighborhood component: The maximum neighborhood component (MNC) and density of maximum neigh-borhood component (DMNC) properties were proposed by Lin et $\mathrm{al}^{26}$ and Chin. ${ }^{4}$ For a protein $i$, let $N(i)$ be the set of neighbors of $i$. The MNC of $i$ is the connected component of $N(i)$ with maximum size, and it is denoted as $M N(i)$.

For a protein $i$, the number of proteins and the number of edges in $M N(i)$ are denoted as $n_{i}$ and $d_{i}$, respectively. DMNC of $i$ is $d_{i} / n_{i}^{\alpha}$ for some $1 \leq$ $\alpha \leq 2$. In their system, they set $\alpha$ to 1.7 .

h. Sequence features: We used ten feature sets from Lin et al. ${ }^{20}$ Let $L$ be the protein length, $F_{i}$ be the occurrence number of amino acid $i$ in the protein, $H(i)$ be the hydrophobic coefficient ${ }^{38}$ of amino acid $i, P_{i, j}$ be the position of the $j$ th occurrence of amino acid $i, A(j)$ be the amino acid of position $j$ and $S_{m, n}$ be the score of row $m$ and column $n$ in PSSM. ${ }^{39}$ The sequence features are listed as follows.

1. Protein length: $L$

2. Cysteine count: $F_{C}$

3. Amino acid occurrence: The composition of amino acid $i$ is $F_{i} / L$, where $1 \leq i \leq 20$.

4. Average cysteine position: $\sum_{j=1}^{F_{C}} P_{C, j} / F_{C}$.

5. Average distance of every two cysteines:

$$
\frac{\sum_{x=1}^{F_{C}} \sum_{y=1}^{F_{C}}\left|P_{C, x}-P_{C, y}\right|}{L \times F_{C}}
$$


6. Cysteine odd-even index: $F_{C} \bmod 2$.

7. Average hydrophobicity: $\sum_{i=1}^{20} F_{i} \times H(i) / L$

8. Average hydrophobicity around cysteine: The $k$ th values of average hydrophobicity around all cysteines were defined as $\sum_{j=1}^{F_{C}} H\left(A\left(P_{C, j}+k\right)\right) / F_{C}$. Here we set $k=-2$, $-1,+1,+2$.

9. Cysteine position distribution: For $1 \leq d \leq \rho$, the $d$ th cysteine position distribution was

$$
\frac{\left|\left\{\alpha_{j} \mid \alpha_{j}=\frac{P_{C, j}}{L}, \frac{d-1}{\rho}<\alpha_{j} \leq \frac{d}{\rho}, 1 \leq j \leq F_{C}\right\}\right|}{F_{c}}
$$

We set $\rho=5$

10. Average PSSM of amino acid: The average PSSM of residue $i$ is $\sum_{m=1}^{L} S_{m, i} / L$.

i. Phyletic retention: Gustafson et $\mathrm{al}^{21}$ discovered that the essential proteins are generally more conserved than nonessential proteins. Phyletic retention of protein $i$ is the number of organisms in which an ortholog is present. The ortholog of each protein was obtained from Hwang et al. ${ }^{7}$

j. Essential index: ${ }^{7}$ Essential index measures the ratio of essential proteins in the neighbors $N(i)$ of node $i$. Essential index of node $i$ is defined as $p(i) / d_{i}$, where $p(i)$ is the number of essential proteins in $N(i)$ and $d_{i}$ is the degree of node $i$.

k. Clique level: ${ }^{7}$ The clique level of protein $i$ is defined as the maximal clique containing $i$. Here, only cliques with sizes between 3 and 10 were taken into consideration.

1. Number of paralagous genes: It is shown that genes are more likely to be essential if there no duplicate existed in the same genome. ${ }^{21}$ This feature is defined as the number of genes that are present in the same genome. In addition, their BLASTP E-values must be less than $10^{-20}$ and the ratios of the larger gene to the smaller do not exceed 1.33. $\mathrm{m}$. Open reading frame length: Gustafson et $\mathrm{al}^{21}$ observed that ancestral genes are more likely to be essential and that proteins generally become larger throughout evolution. Consequently, The open reading frame length may indicate essentiality.

\section{Confidence intervals of performance measures and informational odds ratios}

All performance measures were multiplied by 100. The confidence intervals were set at $95 \%$.
We used the informational odds ratios (IOR) ${ }^{32}$ to represent the association between the essentiality and predictions. IOR measures how much more likely a protein is to be essential when one learning machine outputs essentiality rather than nonessentiality. A value of 1.0 indicates no association between the essentiality and predictions produced by learning machines. All confidence intervals of performance measures and informational odds ratios corresponding to each prediction models are shown in Tables 12-15.

\section{Comparison with other feature selection methods}

We first introduced two feature selection methods that served as benchmarks, $\mathrm{mRMR}^{13}$ and CMIM,${ }^{14}$ both of which are theoretical methods. Next, we compared them with our feature selection method when the feature subsets of equal size were selected.

Unlike other methods that select top-ranking features based on F-score or mutual information without considering relationships among features, mRMR accommodates both feature relevance with respect to class label and dependency among selected features. The strategy combines both the maximal relevance and the minimal redundancy criteria. In order to take the above two criteria into consideration and to avoid an exhaustive search, mRMR adopts an incremental search approach. That is, the $r$ th selected feature should satisfy

$$
X_{r}=\underset{X_{j} \in X-X_{r-1}}{\arg \max }\left\{I\left(X_{j}, Y\right)-\frac{1}{r-1} \sum_{X_{i} \in X_{r-1}} I\left(X_{j}, X_{i}\right)\right\}
$$

where $X$ is the full feature set. $X_{i}$ is a feature within $X_{r-1}$ and $r-1$ is the number of selected features contained in $X_{r-1} . X_{j}$ is any feature that is not yet selected. $I(X, Y)$ is mutual information and it quantifies the dependence (or relevance) between the feature $X_{j}$ and class label $Y$. This means that the $r$ th selected feature should be as relevant to the class label as possible while possesses least dependency averagely to the selected features.

For CMIM, a feature $X_{j}$ is good if $I\left(Y, X_{j} \mid X\right)$ is large for every selected feature $X_{i} . I\left(Y, X_{j} \mid X_{i}\right)$ is conditional mutual information and it quantifies 
Table 12. Confidence intervals of performance measures $(\times 100)$ and informational odds ratios for models produced by the imbalanced S. cerevisiae data set.

\begin{tabular}{lllllll}
\hline & AUC & Precision & Recall & F1 & MCC & IOR \\
\hline CMIM32 & $82.5 \pm 1.2$ & $74.4 \pm 3.1$ & $36.9 \pm 4.5$ & $49.3 \pm 3.8$ & $45.0 \pm 3.6$ & $5.2 \pm 0.5$ \\
mRMR31 & $82.1 \pm 1.6$ & $73.8 \pm 3.2$ & $37.2 \pm 4.3$ & $49.5 \pm 3.6$ & $44.9 \pm 3.4$ & $5.2 \pm 0.5$ \\
Hwang & $77.5 \pm 2.2$ & $74.3 \pm 3.7$ & $34.3 \pm 4.3$ & $46.9 \pm 4.0$ & $43.2 \pm 3.6$ & $5.1 \pm 0.4$ \\
Acencio & $70.7 \pm 3.4$ & $67.5 \pm 6.3$ & $12.1 \pm 5.5$ & $20.4 \pm 7.6$ & $22.8 \pm 6.0$ & $3.7 \pm 0.4$ \\
N4 & $74.4 \pm 2.7$ & $78.2 \pm 3.7$ & $32.7 \pm 4.1$ & $46.1 \pm 4.1$ & $43.9 \pm 3.5$ & $5.3 \pm 0.4$ \\
N5 & $72.7 \pm 3.6$ & $74.1 \pm 4.1$ & $38.7 \pm 4.7$ & $50.9 \pm 4.1$ & $46.1 \pm 3.8$ & $5.3 \pm 0.5$ \\
N6 & $73.0 \pm 3.2$ & $75.2 \pm 4.2$ & $39.5 \pm 4.4$ & $51.8 \pm 3.8$ & $47.2 \pm 3.6$ & $5.5 \pm 0.5$ \\
N7 & $76.1 \pm 2.4$ & $76.7 \pm 3.7$ & $38.6 \pm 4.4$ & $51.3 \pm 3.9$ & $47.3 \pm 3.6$ & $5.5 \pm 0.5$ \\
N8 & $77.2 \pm 2.4$ & $75.5 \pm 3.4$ & $37.1 \pm 4.9$ & $49.8 \pm 4.3$ & $45.7 \pm 3.9$ & $5.3 \pm 0.5$ \\
N9 & $78.2 \pm 2.4$ & $74.9 \pm 3.4$ & $38.2 \pm 4.5$ & $50.6 \pm 3.9$ & $46.2 \pm 3.6$ & $5.4 \pm 0.5$ \\
N10 & $78.1 \pm 2.2$ & $75.1 \pm 3.5$ & $39.9 \pm 4.1$ & $52.1 \pm 3.6$ & $47.4 \pm 3.5$ & $5.5 \pm 0.5$ \\
N11 & $78.6 \pm 2.1$ & $75.2 \pm 3.2$ & $40.2 \pm 4.2$ & $52.4 \pm 3.6$ & $47.6 \pm 3.4$ & $5.5 \pm 0.5$ \\
N12 & $79.8 \pm 2.0$ & $75.9 \pm 3.2$ & $40.9 \pm 4.2$ & $53.2 \pm 3.6$ & $48.5 \pm 3.4$ & $5.7 \pm 0.5$ \\
N13 & $78.9 \pm 1.9$ & $74.8 \pm 3.2$ & $43.3 \pm 4.3$ & $54.9 \pm 3.4$ & $49.5 \pm 3.4$ & $5.8 \pm 0.5$ \\
N14 & $80.2 \pm 1.8$ & $74.9 \pm 3.2$ & $39.7 \pm 4.3$ & $51.9 \pm 3.5$ & $47.1 \pm 3.4$ & $5.5 \pm 0.5$ \\
N15 & $80.1 \pm 1.9$ & $76.3 \pm 3.3$ & $40.6 \pm 4.2$ & $53.0 \pm 3.5$ & $48.5 \pm 3.5$ & $5.7 \pm 0.5$ \\
N16 & $81.4 \pm 1.7$ & $76.2 \pm 3.2$ & $40.1 \pm 4.6$ & $52.5 \pm 3.8$ & $48.0 \pm 3.6$ & $5.6 \pm 0.5$ \\
N17 & $81.4 \pm 1.7$ & $76.1 \pm 3.3$ & $40.7 \pm 4.5$ & $53.0 \pm 3.8$ & $48.4 \pm 3.6$ & $5.7 \pm 0.5$ \\
N18 & $81.1 \pm 1.8$ & $75.1 \pm 3.2$ & $41.1 \pm 4.3$ & $53.1 \pm 3.6$ & $48.2 \pm 3.5$ & $5.6 \pm 0.5$ \\
N90 & $82.9 \pm 1.0$ & $73.8 \pm 2.8$ & $35.5 \pm 4.5$ & $47.9 \pm 3.6$ & $43.8 \pm 3.4$ & $5.1 \pm 0.4$
\end{tabular}

Table 13. Confidence intervals of performance measures $(\times 100)$ and informational odds ratios for models produced by the balanced S. cerevisiae data set.

\begin{tabular}{lllllll}
\hline & AUC & Precision & Recall & F1 & MCC & IOR \\
\hline CMIM32 & $84.2 \pm 1.5$ & $77.2 \pm 2.2$ & $76.6 \pm 2.9$ & $76.9 \pm 2.1$ & $54.0 \pm 3.9$ & $3.3 \pm 0.4$ \\
mRMR31 & $83.6 \pm 1.6$ & $76.5 \pm 2.4$ & $74.1 \pm 3.0$ & $75.2 \pm 2.1$ & $51.3 \pm 4.1$ & $3.0 \pm 0.3$ \\
Hwang & $82.2 \pm 1.7$ & $77.8 \pm 2.6$ & $72.0 \pm 3.7$ & $74.8 \pm 2.3$ & $51.6 \pm 3.9$ & $3.0 \pm 0.3$ \\
Acencio & $76.8 \pm 2.2$ & $69.6 \pm 2.4$ & $73.4 \pm 4.0$ & $71.4 \pm 2.3$ & $41.4 \pm 4.3$ & $2.5 \pm 0.3$ \\
N4 & $81.1 \pm 1.8$ & $77.7 \pm 2.5$ & $71.6 \pm 3.6$ & $74.5 \pm 2.3$ & $51.2 \pm 3.9$ & $3.0 \pm 0.3$ \\
N5 & $82.4 \pm 1.8$ & $77.8 \pm 2.6$ & $73.5 \pm 3.5$ & $75.6 \pm 2.2$ & $52.7 \pm 4.1$ & $3.1 \pm 0.3$ \\
N6 & $82.7 \pm 1.8$ & $77.8 \pm 2.6$ & $73.9 \pm 3.6$ & $75.8 \pm 2.3$ & $53.0 \pm 4.1$ & $3.1 \pm 0.3$ \\
N7 & $83.1 \pm 1.8$ & $77.9 \pm 2.5$ & $73.3 \pm 3.5$ & $75.5 \pm 2.2$ & $52.6 \pm 4.0$ & $3.1 \pm 0.3$ \\
N8 & $82.6 \pm 1.8$ & $78.6 \pm 2.5$ & $72.1 \pm 3.5$ & $75.2 \pm 2.3$ & $52.7 \pm 4.0$ & $3.1 \pm 0.3$ \\
N9 & $83.3 \pm 1.8$ & $79.1 \pm 2.4$ & $73.5 \pm 3.4$ & $76.2 \pm 2.2$ & $54.1 \pm 3.8$ & $3.2 \pm 0.3$ \\
N10 & $83.4 \pm 1.7$ & $78.9 \pm 2.4$ & $73.6 \pm 3.3$ & $76.1 \pm 2.1$ & $54.0 \pm 3.8$ & $3.2 \pm 0.3$ \\
N11 & $83.1 \pm 1.7$ & $78.4 \pm 2.4$ & $73.7 \pm 3.5$ & $76.0 \pm 2.2$ & $53.5 \pm 3.8$ & $3.2 \pm 0.3$ \\
N12 & $82.9 \pm 1.8$ & $77.9 \pm 2.5$ & $73.2 \pm 3.1$ & $75.5 \pm 2.2$ & $52.6 \pm 4.2$ & $3.1 \pm 0.3$ \\
N13 & $83.4 \pm 1.7$ & $78.8 \pm 2.4$ & $73.0 \pm 3.5$ & $75.8 \pm 2.2$ & $53.5 \pm 3.9$ & $3.1 \pm 0.3$ \\
N14 & $83.6 \pm 1.6$ & $77.7 \pm 2.3$ & $74.3 \pm 3.4$ & $75.9 \pm 2.1$ & $53.0 \pm 3.8$ & $3.2 \pm 0.3$ \\
N15 & $84.3 \pm 1.7$ & $78.4 \pm 2.4$ & $74.8 \pm 3.3$ & $76.6 \pm 2.1$ & $54.2 \pm 3.9$ & $3.3 \pm 0.4$ \\
N16 & $84.2 \pm 1.6$ & $77.7 \pm 2.2$ & $75.6 \pm 3.1$ & $76.7 \pm 2.0$ & $54.0 \pm 3.7$ & $3.3 \pm 0.4$ \\
N17 & $84.7 \pm 1.6$ & $77.8 \pm 2.3$ & $76.3 \pm 3.0$ & $77.0 \pm 2.0$ & $54.5 \pm 3.8$ & $3.3 \pm 0.4$ \\
N18 & $84.0 \pm 1.6$ & $77.9 \pm 2.4$ & $74.0 \pm 3.3$ & $75.9 \pm 2.0$ & $53.1 \pm 3.8$ & $3.1 \pm 0.3$ \\
N90 & $83.9 \pm 1.4$ & $76.0 \pm 2.0$ & $75.3 \pm 2.7$ & $75.7 \pm 1.8$ & $51.6 \pm 3.5$ & $3.1 \pm 0.3$ \\
\hline
\end{tabular}


Table 14. Confidence intervals of performance measures $(\times 100)$ and informational odds ratios for models produced by the imbalanced E. coli data set.

\begin{tabular}{lllllll}
\hline & AUC & Precision & Recall & F1 & MCC & IOR \\
\hline CMIM09 & $70.1 \pm 0.9$ & $72.0 \pm 1.4$ & $27.1 \pm 0.7$ & $39.4 \pm 0.9$ & $38.2 \pm 0.9$ & $5.2 \pm 0.6$ \\
mRMR13 & $71.5 \pm 2.4$ & $71.3 \pm 5.4$ & $25.0 \pm 5.8$ & $37.0 \pm 6.8$ & $36.0 \pm 5.8$ & $4.7 \pm 0.6$ \\
Gustafson & $71.1 \pm 2.3$ & $66.5 \pm 4.6$ & $25.5 \pm 5.0$ & $36.8 \pm 5.2$ & $34.7 \pm 4.8$ & $4.9 \pm 0.6$ \\
N4 & $69.1 \pm 1.8$ & $72.5 \pm 1.0$ & $28.0 \pm 0.6$ & $40.4 \pm 0.7$ & $39.1 \pm 0.7$ & $5.5 \pm 0.6$ \\
N5 & $69.0 \pm 2.0$ & $73.7 \pm 1.4$ & $29.5 \pm 0.8$ & $42.1 \pm 0.9$ & $40.7 \pm 1.0$ & $5.7 \pm 0.6$ \\
N6 & $70.1 \pm 1.7$ & $74.2 \pm 1.4$ & $28.7 \pm 0.9$ & $41.4 \pm 1.1$ & $40.3 \pm 1.0$ & $5.7 \pm 0.6$ \\
N7 & $71.4 \pm 1.4$ & $73.5 \pm 1.3$ & $27.5 \pm 0.7$ & $40.0 \pm 0.9$ & $39.2 \pm 0.9$ & $5.5 \pm 0.6$ \\
N8 & $70.5 \pm 1.3$ & $74.2 \pm 1.1$ & $28.8 \pm 0.8$ & $41.5 \pm 0.9$ & $40.5 \pm 0.9$ & $5.7 \pm 0.6$ \\
N9 & $70.7 \pm 1.5$ & $72.6 \pm 1.4$ & $29.3 \pm 1.0$ & $41.7 \pm 1.2$ & $40.1 \pm 1.2$ & $5.6 \pm 0.6$ \\
N10 & $71.1 \pm 1.5$ & $72.4 \pm 1.6$ & $29.4 \pm 1.0$ & $41.8 \pm 1.1$ & $40.1 \pm 1.2$ & $5.6 \pm 0.6$ \\
N11 & $71.4 \pm 1.4$ & $73.2 \pm 1.3$ & $27.8 \pm 0.8$ & $40.3 \pm 1.0$ & $39.3 \pm 1.0$ & $5.5 \pm 0.6$ \\
N12 & $71.2 \pm 1.4$ & $72.5 \pm 1.9$ & $29.2 \pm 1.1$ & $41.6 \pm 1.2$ & $40.0 \pm 1.3$ & $5.6 \pm 0.6$ \\
N13 & $71.4 \pm 1.3$ & $73.3 \pm 1.8$ & $28.7 \pm 1.2$ & $41.3 \pm 1.4$ & $40.0 \pm 1.4$ & $5.6 \pm 0.6$ \\
N80 & $71.6 \pm 0.9$ & $67.7 \pm 2.1$ & $23.7 \pm 1.3$ & $35.2 \pm 1.6$ & $33.9 \pm 1.6$ & $4.9 \pm 0.6$ \\
\hline
\end{tabular}

Table 15. Confidence intervals of performance measures $(\times 100)$ and informational odds ratios for models produced by balanced E. coli data set.

\begin{tabular}{lllllll}
\hline & AUC & Precision & Recall & F1 & MCC & IOR \\
\hline CMIM09 & $76.7 \pm 1.7$ & $72.0 \pm 2.4$ & $70.0 \pm 3.7$ & $71.0 \pm 2.2$ & $42.1 \pm 4.0$ & $2.4 \pm 0.3$ \\
mRMR13 & $76.2 \pm 2.0$ & $72.8 \pm 3.1$ & $65.4 \pm 6.4$ & $68.9 \pm 3.3$ & $39.6 \pm 3.9$ & $2.2 \pm 0.2$ \\
Gustafson & $77.7 \pm 2.6$ & $72.2 \pm 3.3$ & $71.5 \pm 4.0$ & $71.9 \pm 2.8$ & $44.0 \pm 5.5$ & $2.6 \pm 0.3$ \\
N4 & $78.0 \pm 1.6$ & $73.3 \pm 2.5$ & $70.1 \pm 2.7$ & $71.7 \pm 1.9$ & $44.6 \pm 3.8$ & $2.6 \pm 0.3$ \\
N5 & $77.9 \pm 1.7$ & $73.0 \pm 2.5$ & $70.6 \pm 2.8$ & $71.8 \pm 1.8$ & $44.5 \pm 3.8$ & $2.6 \pm 0.3$ \\
N6 & $76.2 \pm 1.7$ & $73.5 \pm 2.6$ & $66.3 \pm 4.3$ & $69.6 \pm 2.6$ & $42.5 \pm 4.1$ & $2.4 \pm 0.3$ \\
N7 & $78.3 \pm 1.7$ & $73.7 \pm 2.5$ & $69.6 \pm 2.7$ & $71.6 \pm 1.8$ & $44.8 \pm 3.6$ & $2.6 \pm 0.3$ \\
N8 & $78.1 \pm 1.7$ & $72.3 \pm 2.3$ & $71.1 \pm 3.3$ & $71.7 \pm 2.1$ & $43.9 \pm 3.8$ & $2.5 \pm 0.3$ \\
N9 & $78.2 \pm 1.6$ & $71.5 \pm 2.2$ & $70.3 \pm 4.1$ & $70.9 \pm 2.3$ & $42.3 \pm 3.8$ & $2.4 \pm 0.3$ \\
N10 & $78.1 \pm 1.6$ & $72.5 \pm 2.4$ & $70.2 \pm 3.3$ & $71.3 \pm 2.1$ & $43.6 \pm 3.9$ & $2.5 \pm 0.3$ \\
N11 & $77.7 \pm 1.7$ & $71.9 \pm 2.2$ & $70.0 \pm 3.3$ & $70.9 \pm 2.0$ & $42.6 \pm 3.6$ & $2.5 \pm 0.3$ \\
N12 & $77.6 \pm 1.9$ & $71.5 \pm 2.3$ & $69.5 \pm 4.5$ & $70.5 \pm 2.5$ & $41.8 \pm 4.0$ & $2.4 \pm 0.3$ \\
N13 & $77.6 \pm 1.7$ & $73.1 \pm 2.4$ & $69.5 \pm 3.0$ & $71.2 \pm 2.1$ & $43.9 \pm 3.9$ & $2.5 \pm 0.3$ \\
N80 & $76.9 \pm 1.8$ & $71.1 \pm 2.4$ & $71.5 \pm 2.4$ & $71.3 \pm 1.8$ & $42.4 \pm 3.8$ & $2.5 \pm 0.3$ \\
\hline
\end{tabular}

Table 16. Performance comparison of our method vs. mRMR for the imbalanced S. cerevisiae data set with the same sizes of feature subsets, where the $>$ symbol represents that the values are significantly higher.

\begin{tabular}{|c|c|c|c|c|c|c|}
\hline & AUC & Precis & & Recall & F-measure & MCC \\
\hline N4 & 0.744 & 0.782 & 0.756 & 0.327 & 0.461 & 0.439 \\
\hline N5 & 0.727 & 0.741 & 0.740 & $0.387 \quad 0.359$ & $0.509 \quad 0.484$ & $0.461 \quad 0.442$ \\
\hline N6 & 0.753 & 0.752 & 0.753 & $0.395>0.333$ & $0.518>0.462$ & $0.472>0.430$ \\
\hline N7 & $0.761 \quad 0.763$ & 0.767 & 0.761 & $0.386>0.330$ & $0.513>0.460$ & $0.473>0.431$ \\
\hline N8 & $0.772>0.771$ & 0.755 & 0.757 & $0.371>0.326$ & $0.498>0.456$ & $0.457>0.427$ \\
\hline N9 & $0.782 \quad 0.776$ & 0.749 & 0.749 & $0.382>0.341$ & $0.506>0.469$ & $0.462>0.434$ \\
\hline N10 & $0.781>0.778$ & 0.751 & 0.752 & $0.399>0.340$ & $0.521>0.469$ & $0.474>0.434$ \\
\hline N11 & $0.786>0.774$ & 0.752 & 0.750 & $0.402>0.341$ & $0.524>0.469$ & $0.476>0.434$ \\
\hline N12 & $0.798>0.781$ & 0.759 & 0.757 & $0.409>0.334$ & $0.532>0.463$ & $0.485>0.432$ \\
\hline N13 & $0.789>0.774$ & 0.748 & 0.746 & $0.433>0.342$ & $0.549>0.469$ & $0.495>0.432$ \\
\hline N14 & $0.802>0.775$ & 0.749 & 0.750 & $0.397>0.340$ & $0.519>0.468$ & $0.471>0.433$ \\
\hline N15 & $0.801>0.798$ & 0.763 & 0.764 & $0.406>0.318$ & $0.530>0.449$ & $0.485>0.424$ \\
\hline N16 & $0.814>0.799$ & 0.762 & 0.762 & $0.401>0.318$ & $0.525>0.449$ & $0.480>0.423$ \\
\hline N17 & $0.814>0.799$ & 0.761 & 0.759 & $0.407>0.326$ & $0.530>0.456$ & $0.484>0.427$ \\
\hline N18 & $0.811>0.797$ & 0.751 & 0.749 & $0.411>0.342$ & $0.531>0.469$ & $0.482>0.434$ \\
\hline
\end{tabular}


Table 17. Performance comparison of our new method vs. CMIM for the imbalanced S. cerevisiae data set when identical number of features are selected.

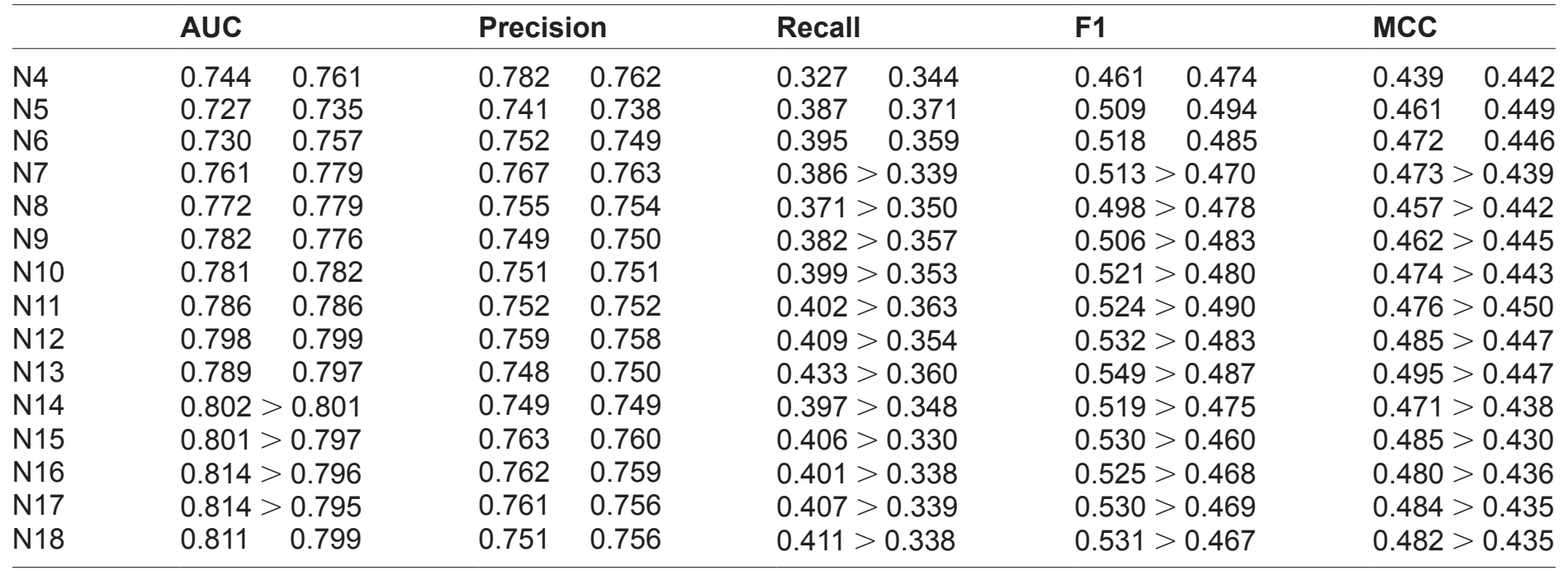

discrepancy between features $X_{j}$ and $X_{i}$ for given the class label $Y$. Consequently, the feature selection procedure was also carried out in an incremental manner as follows:

$$
\begin{gathered}
v(1)=\arg \max _{X_{j}} I\left(X_{j}, Y\right) \\
v(r)=\arg \max _{X_{j}}\left\{\min _{k \leq r-1} I\left(Y, X_{j} \mid X_{v(k)}\right)\right\} .
\end{gathered}
$$

where $v(k)$ denotes the $k$ th selected feature.
In the following paragraph, we compare our method with the above two feature selection methods when the feature subsets of equal size were selected. We first ran the SVM software with Hwang's or Gustafson feature sets and tune the SVM parameters to achieve the highest average performances. To fairly compare the methods given feature subsets with same sizes obtained by our methods, mRMR and CMIM, we used the same SVM software and adjust the cost parameters in order to achieve similar levels of precision. For $S$. cerevisiae and $E$. coli data set, the feature numbers $k$ are $4 \leq k \leq 18$ and

Table 18. Performance comparison of our method vs. mRMR for the balanced $S$. cerevisiae data set with the same sizes

\begin{tabular}{|c|c|c|c|c|c|c|c|c|c|}
\hline \multirow[b]{2}{*}{ N4 } & AUC & \multicolumn{2}{|c|}{ Precision } & \multicolumn{2}{|l|}{ Recall } & \multicolumn{2}{|c|}{ F-measure } & \multicolumn{2}{|l|}{ MCC } \\
\hline & 0.815 & 0.777 & 0.770 & 0.716 & 0.725 & 0.745 & 0.747 & 0.512 & 0.510 \\
\hline N5 & 0.818 & 0.778 & 0.771 & 0.735 & 0.722 & 0.756 & 0.745 & 0.527 & 0.508 \\
\hline N6 & 0.827 & 0.778 & 0.775 & 0.739 & 0.709 & 0.758 & 0.740 & 0.530 & 0.504 \\
\hline N7 & 0.831 & 0.779 & 0.779 & 0.733 & 0.718 & 0.755 & 0.747 & 0.526 & 0.516 \\
\hline N8 & 0.826 & 0.786 & 0.781 & 0.721 & 0.721 & 0.752 & 0.750 & 0.527 & 0.521 \\
\hline N9 & 0.833 & 0.791 & 0.783 & 0.735 & 0.734 & 0.762 & 0.758 & 0.541 & 0.531 \\
\hline N10 & 0.835 & 0.789 & 0.783 & 0.736 & 0.733 & 0.761 & 0.757 & 0.540 & 0.531 \\
\hline N11 & 0.831 & 0.784 & 0.780 & 0.737 & 0.730 & 0.760 & 0.754 & 0.535 & 0.525 \\
\hline N12 & 0.829 & 0.779 & 0.778 & 0.732 & 0.734 & 0.755 & 0.755 & 0.526 & 0.525 \\
\hline N13 & 0.834 & 0.788 & 0.779 & 0.730 & 0.732 & 0.758 & 0.754 & 0.535 & 0.525 \\
\hline N14 & 0.832 & 0.777 & 0.777 & 0.743 & 0.731 & 0.759 & 0.753 & 0.530 & 0.522 \\
\hline N15 & 0.835 & 0.784 & 0.778 & 0.748 & 0.734 & 0.766 & 0.756 & 0.542 & 0.526 \\
\hline N16 & $0.842>0.836$ & 0.777 & 0.777 & $0.756>$ & 0.735 & 0.767 & 0.755 & 0.540 & 0.525 \\
\hline N17 & $0.847>0.834$ & 0.778 & 0.777 & 0.763 & 0.733 & 0.770 & 0.754 & 0.545 & 0.523 \\
\hline N18 & $0.840 \quad 0.835$ & 0.779 & 0.778 & 0.740 & 0.735 & 0.759 & 0.756 & 0.531 & 0.526 \\
\hline
\end{tabular}
of feature subsets, where the $>$ symbol indicates that the values are significantly higher. 
Table 19. Performance comparison of our method vs. CMIM for the balanced S. cerevisiae data set when identical number of features are selected.

\begin{tabular}{|c|c|c|c|c|c|c|c|c|c|}
\hline \multirow[b]{2}{*}{ N4 } & AUC & \multicolumn{2}{|c|}{ Precision } & \multicolumn{2}{|l|}{ Recall } & \multicolumn{2}{|l|}{ F1 } & \multicolumn{2}{|l|}{ MCC } \\
\hline & 0.813 & 0.777 & 0.777 & 0.716 & 0.724 & 0.745 & 0.749 & 0.512 & 0.517 \\
\hline N5 & 0.817 & 0.778 & 0.775 & 0.735 & 0.740 & 0.756 & 0.757 & 0.527 & 0.526 \\
\hline N6 & 0.821 & 0.778 & 0.777 & 0.739 & 0.742 & 0.758 & 0.759 & 0.530 & 0.529 \\
\hline N7 & 0.830 & 0.779 & 0.772 & 0.733 & 0.744 & 0.755 & 0.758 & 0.526 & 0.524 \\
\hline N8 & 0.833 & 0.786 & 0.776 & 0.721 & 0.738 & 0.752 & 0.756 & 0.527 & 0.525 \\
\hline N9 & 0.834 & 0.791 & 0.775 & 0.735 & 0.740 & 0.762 & 0.757 & 0.541 & 0.526 \\
\hline N10 & 0.835 & 0.789 & 0.776 & 0.736 & 0.739 & 0.761 & 0.757 & 0.540 & 0.527 \\
\hline N11 & 0.836 & 0.784 & 0.778 & 0.737 & 0.739 & 0.760 & 0.758 & 0.535 & 0.528 \\
\hline N12 & 0.838 & 0.779 & 0.779 & 0.732 & 0.742 & 0.755 & 0.760 & 0.526 & 0.532 \\
\hline N13 & 0.837 & 0.788 & 0.778 & 0.730 & 0.741 & 0.758 & 0.759 & 0.535 & 0.530 \\
\hline N14 & 0.836 & 0.777 & 0.777 & 0.743 & 0.743 & 0.759 & 0.759 & 0.530 & 0.530 \\
\hline N15 & 0.843 & 0.784 & 0.777 & 0.748 & 0.739 & 0.766 & 0.758 & 0.542 & 0.528 \\
\hline N16 & $0.842>0.837$ & 0.777 & 0.776 & 0.756 & 0.741 & 0.767 & 0.758 & 0.540 & 0.528 \\
\hline N17 & $0.847>0.838$ & 0.778 & 0.777 & $0.763>$ & 0.744 & 0.770 & 0.760 & 0.545 & 0.531 \\
\hline N18 & $0.840 \quad 0.837$ & 0.779 & 0.778 & 0.740 & 0.746 & 0.759 & 0.762 & 0.531 & 0.533 \\
\hline
\end{tabular}

Table 20. Performance comparison of our method vs. mRMR for the imbalanced $E$. coli data set when identical numbers of features are selected.

\begin{tabular}{|c|c|c|c|c|c|c|c|c|c|c|}
\hline \multirow[b]{2}{*}{ N4 } & \multicolumn{2}{|l|}{ AUC } & \multicolumn{2}{|c|}{ Precision } & \multicolumn{2}{|c|}{ Recall } & \multicolumn{2}{|l|}{ F1 } & \multicolumn{2}{|l|}{ MCC } \\
\hline & \multirow{2}{*}{0.691} & 0.651 & \multirow{2}{*}{$\begin{array}{l}0.725 \\
0.737\end{array}$} & 0.678 & \multirow{2}{*}{$\begin{array}{l}0.280 \\
0.295\end{array}$} & 0.269 & 0.404 & 0.385 & \multirow{2}{*}{0.391} & \multirow{2}{*}{0.363} \\
\hline N5 & & 0.675 & & 0.687 & & 0.254 & 0.421 & 0.371 & & \\
\hline N6 & 0.701 & 0.681 & 0.742 & 0.708 & 0.287 & 0.220 & 0.414 & 0.336 & 0.403 & 0.338 \\
\hline N7 & 0.714 & 0.686 & 0.735 & 0.712 & 0.275 & 0.212 & 0.400 & 0.326 & 0.392 & 0.333 \\
\hline N8 & 0.705 & 0.692 & 0.742 & 0.713 & 0.288 & 0.209 & 0.415 & 0.323 & 0.405 & 0.330 \\
\hline N9 & 0.707 & 0.692 & 0.726 & 0.713 & 0.293 & 0.199 & $0.417>$ & 0.312 & 0.401 & 0.322 \\
\hline N10 & 0.711 & 0.697 & 0.724 & 0.703 & 0.294 & 0.193 & $0.418=$ & 0.302 & 0.401 & 0.313 \\
\hline N11 & 0.714 & 0.702 & 0.732 & 0.697 & 0.278 & 0.187 & 0.403 & 0.295 & 0.393 & 0.306 \\
\hline N12 & 0.712 & 0.704 & 0.725 & 0.683 & 0.292 & 0.192 & 0.416 & 0.300 & 0.400 & 0.305 \\
\hline N13 & 0.714 & 0.715 & 0.733 & 0.713 & 0.287 & 0.250 & 0.413 & 0.370 & 0.400 & 0.360 \\
\hline
\end{tabular}

Table 21. Performance comparison of our method vs. CMIM for the imbalanced E. coli data set when identical numbers of features are selected.

\begin{tabular}{|c|c|c|c|c|c|c|}
\hline \multirow[b]{2}{*}{ N4 } & AUC & \multicolumn{2}{|c|}{ Precision } & Recall & F1 & MCC \\
\hline & $0.691>0.663$ & 0.725 & 0.717 & $0.280 \quad 0.271$ & 0.404 & 0.391 \\
\hline N5 & $0.690 \quad 0.686$ & 0.737 & 0.710 & $0.295>0.264$ & $0.421>0.385$ & $0.407>0.373$ \\
\hline N6 & 0.697 & 0.742 & 0.715 & $0.287>0.265$ & $0.414>0.387$ & $0.403>0.376$ \\
\hline N7 & 0.693 & 0.735 & 0.711 & $0.275>0.261$ & $0.400>0.382$ & $0.392>0.371$ \\
\hline N8 & 0.690 & 0.742 & 0.709 & $0.288>0.254$ & $0.415>0.373$ & $0.405>0.364$ \\
\hline N9 & 0.701 & 0.726 & 0.720 & $0.293>0.271$ & $0.417>0.394$ & $0.401>0.382$ \\
\hline N10 & 0.702 & 0.724 & 0.692 & $0.294>0.248$ & $0.418>0.364$ & $0.401>0.353$ \\
\hline N11 & 0.698 & 0.732 & 0.690 & $0.278>0.247$ & $0.403>0.363$ & $0.393>0.351$ \\
\hline N12 & 0.690 & 0.725 & 0.683 & $0.292>0.239$ & $0.416>0.353$ & $0.400>0.342$ \\
\hline N13 & 0.688 & 0.733 & 0.678 & $0.287>0.236$ & $0.413>0.349$ & $0.400>0.337$ \\
\hline
\end{tabular}


Table 22. Performance comparison of our method vs. mRMR for the balanced E. coli data set when identical numbers of features are selected.

\begin{tabular}{|c|c|c|c|c|c|c|}
\hline \multirow[b]{2}{*}{ N4 } & \multirow{2}{*}{$\begin{array}{l}\text { AUC } \\
0.780>0.773\end{array}$} & \multicolumn{2}{|c|}{ Precision } & Recall & \multirow{2}{*}{$\begin{array}{l}\text { F1 } \\
0.717>0.686\end{array}$} & \multirow{2}{*}{$\begin{array}{l}\text { MCC } \\
0.446>0.407\end{array}$} \\
\hline & & 0.733 & 0.726 & $0.701>0.651$ & & \\
\hline N5 & $0.779 \quad 0.772$ & 0.730 & 0.720 & $0.706>0.654$ & $0.718>0.684$ & $0.445>0.401$ \\
\hline N6 & 0.762 & 0.735 & 0.717 & $0.663 \quad 0.649$ & $0.696 \quad 0.680$ & $0.425 \quad 0.394$ \\
\hline N7 & $0.783>0.768$ & 0.737 & 0.716 & $0.696 \quad 0.649$ & $0.716>0.680$ & $0.448>0.394$ \\
\hline N8 & $0.781>0.764$ & 0.723 & 0.715 & $0.711>0.641$ & $0.717>0.675$ & $0.439>0.387$ \\
\hline N9 & $0.782>0.764$ & 0.715 & 0.713 & $0.703 \quad 0.643$ & $0.709>0.675$ & $0.423>0.386$ \\
\hline N10 & $0.781>0.765$ & 0.725 & 0.716 & $0.702>0.636$ & $0.713>0.673$ & $0.436>0.386$ \\
\hline N11 & $0.777>0.766$ & 0.719 & 0.720 & $0.700 \quad 0.643$ & $0.709>0.678$ & $0.426 \quad 0.394$ \\
\hline N12 & $0.776 \quad 0.765$ & 0.715 & 0.714 & $0.695 \quad 0.643$ & $0.705 \quad 0.676$ & $0.418 \quad 0.388$ \\
\hline N13 & $0.776>0.762$ & 0.731 & 0.728 & $0.695>0.654$ & $0.712>0.689$ & $0.439>0.396$ \\
\hline
\end{tabular}

$4 \leq k \leq 13$, respectively, to be compliant with those in Section V.

For the S. cerevisiae data set in the imbalanced experiment, Tables 16 and 17 show the performance comparison of our method versus mRMR and CMIM. Our method performed significantly better when the size of a feature subset exceeded 7. For the balanced experiment, as illustrated in Tables 18 and 19, our method was significantly better only when the number of selected features exceeded 16.

For the E. coli data set in the imbalanced experiment, Tables 20 and 21 show the performance comparison of our method versus mRMR and CMIM with configurations similar to the $S$. cerevisiae data set. Our method performed significantly better when the size of a feature subset exceeded 9. For the balanced experiment, as illustrated in Table 22 and 23, our method performed significantly better than mRMR when the numbers of selected features exceeded 7. The experimental results showed almost no difference between our method and CMIM except for N6 AUC.

For methods such as mRMR and CMIM, both relevance and information redundancy are taken into consideration. Therefore, the obtained feature subsets were quite compact as well as effective. However, the relevance may only be appropriate for some performance measures, such as classification accuracy or precision. Our method took both the performance and feature size into consideration. Consequently, the resultant feature subsets were more effective in some other performance measures for given equal number of features and precision values.

Table 23. Performance comparison of our method vs. CMIM for the balanced E. coli data set when identical numbers of features are selected.

\begin{tabular}{llllllllllll}
\hline & AUC & \multicolumn{3}{c}{ Precision } & \multicolumn{2}{c}{ Recall } & & F1 & MCC \\
\hline N4 & 0.780 & 0.769 & 0.733 & 0.719 & 0.701 & 0.696 & 0.717 & 0.707 & 0.446 & 0.424 \\
N5 & 0.779 & 0.771 & 0.730 & 0.715 & 0.706 & 0.696 & 0.718 & 0.705 & 0.445 & 0.419 \\
N6 & 0.762 & 0.771 & 0.735 & 0.716 & 0.663 & 0.684 & 0.696 & 0.699 & 0.425 & 0.413 \\
N7 & 0.783 & 0.769 & 0.737 & 0.711 & 0.696 & 0.696 & 0.716 & 0.703 & 0.448 & 0.413 \\
N8 & 0.781 & 0.767 & 0.723 & 0.709 & 0.711 & 0.697 & 0.717 & 0.703 & 0.439 & 0.412 \\
N9 & 0.782 & 0.767 & 0.715 & 0.720 & 0.703 & 0.700 & 0.709 & 0.710 & 0.423 & 0.421 \\
N10 & 0.781 & 0.767 & 0.725 & 0.705 & 0.702 & 0.702 & 0.713 & 0.704 & 0.436 & 0.409 \\
N11 & 0.777 & 0.765 & 0.719 & 0.706 & 0.700 & 0.700 & 0.709 & 0.703 & 0.426 & 0.408 \\
N12 & 0.776 & 0.764 & 0.715 & 0.703 & 0.695 & 0.698 & 0.705 & 0.700 & 0.418 & 0.404 \\
N13 & 0.776 & 0.765 & 0.731 & 0.704 & 0.695 & 0.700 & 0.712 & 0.702 & 0.439 & 0.406 \\
\hline
\end{tabular}




\section{Author Contributions}

Conceived and designed the experiments: CBY, CYH. Analysed the data: CYH, ZJY. Wrote the first draft of the manuscript: CYH, ZJY, CTT. Contributed to the writing of the manuscript: CYH, CBY, ZJY. Agree with manuscript results and conclusions: $\mathrm{CYH}, \mathrm{CBY}$. Jointly developed the structure and arguments for the paper: CYH, CBY, ZJY, CTT. Made critical revisions and approved final version: CBY. All authors reviewed and approved the final manuscript.

\section{Funding}

This research work was partially supported by the National Science Council of Taiwan under contract NSC100-2221-E-110-050.

\section{Competing Interests}

Author(s) disclose no potential conflicts of interest.

\section{Disclosures and Ethics}

As a requirement of publication the authors have provided signed confirmation of their compliance with ethical and legal obligations including but not limited to compliance with ICMJE authorship and competing interests guidelines, that the article is neither under consideration for publication nor published elsewhere, of their compliance with legal and ethical guidelines concerning human and animal research participants (if applicable), and that permission has been obtained for reproduction of any copyrighted material. This article was subject to blind, independent, expert peer review. The reviewers reported no competing interests. A preliminary version of this paper was presented at the International Conference on Machine Learning and Applications. ${ }^{40}$

\section{References}

1. Cadigan KM, Grossniklaus U, Gehring WJ. Functional redundancy: The respective roles of the two sloppy paired genes in drosophila segmentation. Proc Natl Acad Sci U S A. 1994;91(14):6324-8.

2. Cullen LM, Arndt GM. Genome-wide screening for gene function using RNAi in mammalian cells. Immunol Cell Biol. 2005;83(3):217-23.

3. Roemer T, Jiang B, Davison J, et al. Large-scale essential gene identification in candida albicans and applications to antifungal drug discovery. Mol Microbiol. 2003;50(1):167-81.

4. Chin CH. Prediction of Essential Proteins and Functional Modules From Protein-Protein Interaction Networks. dissertation, [dissertation]. National Central University, Chung-Li, Taiwan, 2010.

5. Acencio ML, Lemke N. Towards the prediction of essential genes by integration of network topology, cellular localization and biological process information. BMC Bioinformatics. 2009;10:290.
6. Witten IH, Frank E, Hall MA. Data Mining:Practical Machine Learning Tools and Techniques with Java Implementations. Burlington, MA: Morgann Kaufmann; 2000.

7. Hwang YC, Lin CC, Chang JY, Mori H, Juan HF, Huang HC. Predicting essential genes based on network and sequence analysis. Mol Biosyst. 2009; 5(12):1672-8.

8. Przulj N, Wigle DA, Jurisica I. Functional topology in a network of protein interactions. Bioinformatics. 1998;20(3):340-8.

9. Chin CS, Samanta MP. Global snapshot of a protein interaction network percolation based approach. Bioinformatics. 2003;19(18):2413-9.

10. Barabási AL, Oltvai ZN. Network biology: understanding the cell's functional organization. Nat Rev Genet. 2004;5(2):101-13.

11. Ito T, Chiba T, Ozawa R, Yoshida M, Hattori M, Sakaki Y. A comprehensive two-hybrid analysis to explore the yeast protein interactome. Proc Natl Acad Sci U S A. 2001;98(8):4569-74.

12. Lal TN, Chapelle O, Schölkopf B. Feature Extraction: Foundations and Applications. Guyon S, Gunn M, Nikravesh M, Zadeh LA. Berlin: Springer; 2006.

13. Peng H, Long F, Ding C. Feature selection based on mutual information: criteria of max-dependency, max-relevance, and min-redundancy. IEEE Trans Pattern Anal Mach Intell. 2005;27(8):1226-38.

14. Fleuret F. Fast binary feature selection with conditional mutual information. Journal of Machine Learning Research. 2004;5:1531-55.

15. Sotoca JM, Pla SF. Supervised feature selection by clustering using conditional mutual information-based distances. Pattern Recognition. 2010;43(6): 2068-81.

16. Quinlan JR. C4.5: Programs for Machine Learning. Morgan Kaufmann: Burlington, MA; 1993.

17. Breiman L, Friedman J, Stone CJ, Olshen RA. Classification and Regression Trees. London: Chapman and Hall/CRC; 1984.

18. Tan PN, Steinbach M, Kumar V. Introduction to Data Mining. Boston, MA: Addison-Wesley; 2005.

19. Wang G, Ma J, Yan S. IGF-bagging: Information gain based feature selection for bagging. International Journal of Innovative Computing, Information and Control. 2011;7(11):6247-59.

20. Lin CY, Yang CB, Hor CY, Huang KS. Disulfide bonding state prediction with svm based on protein types. Bio-Inspired Computing: Theories and Applications. 2010:1436-42.

21. Gustafson AM, Snitkin ES, Parker SC, DeLisi C, Kasif S. Towards the identification of essential genes using targeted genome sequencing and comparative analysis. BMC Genomics. 2006;7:265.

22. Hu P, Janga SC, Babu M, et al. Global functional atlas of Escherichia coli encompassing previously uncharacterized proteins. PLoS Biol. 2009;7(4):e96.

23. Chang CC, Lin CJ. LIBSVM: a library for support vector machines. http:// www.csie.ntu.edu.tw/ cjlin/libsvm. Updated date April 1, 2013. Accessed date Feb 1, 2012.

24. Fu WJ, Carroll RJ, Wang S. Estimating misclassification error with small samples via bootstrap cross-validation. Bioinformatics. 2005;21(9):1979-86.

25. Salwinski L, Miller CS, Smith AJ, Pettit FK, Bowie JU, Eisenberg D. The database of interacting proteins: 2004 update. Nucleic Acids Res. 2004;32: D449-51.

26. Lin CY, Chin CH, Wu HH, Chen SH, Ho CW, Ko MT. Hubba: hub objects analyzer - a framework of interactome hubs identification for network biology. Nucleic Acids Res. 2008;36(Web server issue):W438-43.

27. Begum N, Fattah MA, Ren F. Automatic text summarization using support vector machine. International Journal of Innovative Computing, Information and Control. 2009;7(5):1987.

28. Chen RC, Chen SP. Intrusion detection using a hybrid support vector machine based on entropy and TF-IDF. International Journal of Innovative Computing, Information and Control. 2008;4(2):413-24

29. She QS, Su HY, Dong L, Chu J. Support vector machine with adaptive parameters in image coding. International Journal of Innovative Computing, Information and Control. 2008;4(2):359-67.

30. Sing T, Sander O, Beerenwinkel N, Lengauer T. ROCR: visualizing classifier performance in R. Bioinformatics. 2005;21(20):3940-1. 
31. R Development Core Team. R: A Language and Environment for Statistical Computing. Vienna, Austria: R Foundation for Statistical Computing; 2008.

32. Efird JT, Lea S, Toland A, Phillips CJ. Informational odds ratio: a useful measure of epidemiologic association in environment exposure studies. Environ Health Insights. 2012;6:17-25.

33. Demsar J. Statistical comparisons of classifiers over multiple data sets. Journal of Machine Learning Research. 2006;7:1-30.

34. Chang YI, Wu CC, Chen JR, Jeng YH. Mining sequence motifs from protein databases based on a bit pattern approach. International Journal of Innovative Computing, Information and Control. 2012;8(1B):647-57.

35. Ruta D, Gabrys B. A theoretical analysis of the limits of majority voting errors for multiple classifier systems. Pattern Analysis and Applications. 2002;5(4):333-50.

36. Kuncheva L. Measures of diversity in classifier ensembles and their relationship with the ensemble accuracy. Machine Learning. 2003;51(2):181-207.

37. Csárdi G, Nepusz T. The igraph software package for complex network research. InterJournal. 2006; Complex systems:1695.

38. Kyte J, Doolittle RF. A simple method for displaying the hydropathic character of a protein J Mol Biol. 1982;157(1):105-32.
39. Altschul SF, Madden TL, Schäffer AA, et al. Gapped BLAST and PSIBLAST: a new generation of protein database search programs. Nucleic Acids Res. 1997;25(17):3389-402.

40. Hor CY, Yang CB, Yang ZJ, Tseng CT. Prediction of protein essentiality by the support vector machine with statistical tests. 11th International Conference on Machine Learning and Applications; Boca Raton, Florida. 2012: 96-101.

41. Yu H, Kim PM, Sprecher E, Trifonov V, Gerstein M. The importance of bottlenecks in protein networks: correlation with gene essentiality and expression dynamics. PLoS Comput Biol. 2007;3(4):e59.

42. Wuchty S, Stadle PF. Centers of complex networks. J Theor Biol. 2003; 223(1):43-53.

43. Jeong H, Mason SP, Barabási A-L, Oltvai ZN. Lethality and centrality in protein networks. Nature. 2001;411(6833):41-2.

44. Peden J. Analysis of Codon Usage. [dissertation] University of Nottingham, UK, 1999. 